\title{
The isotropic-nematic phase transition of tangent hard-sphere chain fluids-Pure components
}

\author{
Thijs van Westen, ${ }^{1}$ Bernardo Oyarzún, ${ }^{1}$ Thijs J. H. Vlugt, ${ }^{1}$ and Joachim Gross ${ }^{2, a)}$ \\ ${ }^{1}$ Process and Energy Laboratory, Delft University of Technology, Leeghwaterstraat 44, \\ 2628 CA Delft, The Netherlands \\ ${ }^{2}$ Institut für Thermodynamik und Thermische Verfahrenstechnik, Universität Stuttgart, Pfaffenwaldring 9, \\ 70569 Stuttgart, Germany
}

(Received 17 May 2013; accepted 2 July 2013; published online 19 July 2013)

\begin{abstract}
An extension of Onsager's second virial theory is developed to describe the isotropic-nematic phase transition of tangent hard-sphere chain fluids. Flexibility is introduced by the rod-coil model. The effect of chain-flexibility on the second virial coefficient is described using an accurate, analytical approximation for the orientation-dependent pair-excluded volume. The use of this approximation allows for an analytical treatment of intramolecular flexibility by using a single pure-component parameter. Two approaches to approximate the effect of the higher virial coefficients are considered, i.e., the Vega-Lago rescaling and Scaled Particle Theory (SPT). The Onsager trial function is employed to describe the orientational distribution function. Theoretical predictions for the equation of state and orientational order parameter are tested against the results from Monte Carlo (MC) simulations. For linear chains of length 9 and longer, theoretical results are in excellent agreement with MC data. For smaller chain lengths, small errors introduced by the approximation of the higher virial coefficients become apparent, leading to a small under- and overestimation of the pressure and density difference at the phase transition, respectively. For rod-coil fluids of reasonable rigidity, a quantitative comparison between theory and MC simulations is obtained. For more flexible chains, however, both the Vega-Lago rescaling and SPT lead to a small underestimation of the location of the phase transition. () 2013 AIP Publishing LLC. [http://dx.doi.org/10.1063/1.4815916]
\end{abstract}

\section{INTRODUCTION}

The liquid crystalline state is a state of matter in which molecules are arranged with a long-ranged (partial) orientational and/or positional order. The properties of these systems generally vary between those of the isotropic liquid and the perfectly ordered crystal. Systems that can form liquid crystalline phases consist of either low molecular weight organic molecules, ${ }^{1,2}$ solutions of polymer melts or amphiphilic molecules, or even suspensions of inorganic or biological colloidal particles such as $\mathrm{V}_{2} \mathrm{O}_{5},{ }^{3,4}$ bentonite clay platelets, ${ }^{4}$ or viruses, ${ }^{5}$ respectively. Due to their interesting properties, liquid crystals have found numerous applications, ${ }^{1,6,7}$ ranging from food-products, cosmetics, and pharmaceuticals to, probably most evidently, the use in LCDs for electronic devices. Recently, liquid crystals have also been proposed as novel solvents for gas-absorption processes. ${ }^{8,9}$

As already pointed out by Onsager in the early 1940s, a key attribute for the formation of a liquid crystalline phase is anisotropy in the shape of the molecular hard cores. ${ }^{10,11}$ For a system of hard rods, Onsager showed that the orientational ordering transition is completely entropically driven. While at low density the orientational entropy dominates, forcing the molecules in an isotropic (randomly orientated) state, at higher densities there is a compensating translaa) Author to whom correspondence should be addressed. Electronic mail:
gross@itt.uni-stuttgart.de tional entropy/free volume that can be gained by aligning the molecules to minimize the excluded volume. Onsager's formalism can be generalized to many different types of hard anisotropic molecules, ${ }^{12}$ and, as shown by Bolhuis and Frenkel, ${ }^{13}$ it becomes exact in the low density limit (i.e., for molecules of infinite shape anisotropy). Typical model systems that have been studied in this respect, are hard discs, ${ }^{14,15}$ rods, ${ }^{16}$ cut spheres, ${ }^{17,18}$ spherocylinders, ${ }^{13,19}$ ellipsoids, ${ }^{20,21}$ Gaussian overlap particles, ${ }^{22}$ and hard-sphere chains. ${ }^{23-27}$ Despite the simplicity of these molecular models, a rich phase behavior, involving isotropic (iso), nematic (nem), smectic, and columnar phases has been observed both by experiments ${ }^{16}$ and by means of molecular simulations ${ }^{13,17,20,27}$ and theory. ${ }^{12,18,28}$

In the present work, the liquid crystalline state of interest is the nematic state. Here, the distribution of molecules is characterized by partial orientational order and no (longranged) positional order. Although Onsager's original second virial theory and its extensions to higher density ${ }^{29-39}$ (to deal with molecules of only moderate shape anisotropy) have been used successfully to describe nematic ordering of many convex molecular models, ${ }^{18,19,22}$ the application to non-convex models still needs consideration. Especially, the introduction of intramolecular flexibility in these models is problematic, usually involving time-consuming Monte Carlo (MC) simulations to compute ensemble averages of the orientation-dependent pair-excluded volume over all internal degrees of freedom of the chain molecules. ${ }^{23,40}$ The 
introduction of intramolecular flexibility is important, however, since this is a key aspect determining the stability of a nematic phase. . $^{25,26,41,42}$

In this work, molecular flexibility is introduced through the tangent hard-sphere chain model. To model the partial flexibility of real liquid crystal molecules, ${ }^{2}$ one part of the chain is arranged in a rigid, linear conformation while the other part is fully flexible. Onsager's second virial theory is extended to incorporate the effects of intramolecular flexibility using the method of Fynewever and Yethiraj. ${ }^{23}$ In contrast to the original work of Fynewever and Yethiraj, the ensemble average of the pair-excluded volume that is needed to calculate the second virial coefficient is here obtained from an analytical approximation ${ }^{43}$ instead of MC simulations of two chain molecules. Consequently, the resulting theory is computationally much more efficient, while, due to the high accuracy of the approximation, a similar accuracy is maintained. A key result of this new approach is that the flexibility of a molecule is treated analytically and can be described by a single purecomponent parameter. In the spirit of molecular perturbation theory, ${ }^{44,45}$ this result is appealing since intramolecular flexibility can with this approach be treated at the stage of the purely repulsive reference fluid.

To describe the effect of higher virial coefficients, both the Vega-Lago rescaling ${ }^{32}$ and Scaled Particle Theory ${ }^{33-39}$ (SPT) are used. A successful application of the Vega-Lago approach requires an accurate description of the isotropic equation of state (EoS). Inspired by the work of Hino and Prausnitz, ${ }^{46}$ we evaluate the Liu-Hu (LH) $\operatorname{EoS}^{47}$ for this. In addition, a recently developed extension of the LH EoS to partially flexible (rod-coil) and linear hard-sphere chain fluids is tested. ${ }^{48}$ To characterize the orientational distribution function (ODF), we use the hyperbolic trial function of Onsager. As recently shown by Franco-Melgar et al. ${ }^{12}$ for the specific case of a system of hard spherocylinders, the use of the Onsager trial function leads to an almost identical description of the nematic ordering compared to the use of a full numerical solution of the ODF. We show that this observation also holds for our case.

This paper is organized as follows. In Sec. II, the molecular model is presented. In Sec. III, Onsager's second virial theory is extended to partially flexible chain molecules. In addition, the Vega-Lago approach and SPT are discussed. In Sec. IV, theoretical predictions for the equation of state and nematic order parameter are extensively tested to MC simulations from literature. ${ }^{27}$ We summarize our findings in Sec. V.

\section{MOLECULAR MODEL}

The molecular model employed in this work assumes a chain of $m$ tangent hard spheres of diameter $\sigma$. To mimic the structure of "real" liquid crystal molecules, one part of the chain is arranged in a rigid, linear conformation (referred to as "rod") while the other part is completely flexible (referred to as "coil"). This model will be referred to as rod-coil fluid. In the remainder of this paper, we employ a general $m-m_{R}$ notation to denote a rod-coil with a total number of $m$ segments and a number of $m_{R}$ segments in the rigid block. A completely linear or flexible chain is simply referred to as a linear or flexible $m$-mer.

As discussed in a previous work, ${ }^{43}$ it is convenient to introduce a dimensionless rigidity parameter, defined as the ratio of the total number of rigid bond angles and the total number of bond angles in a molecule:

$$
\chi_{R}= \begin{cases}\frac{m_{R}-2}{m-2} & \text { for } m>2 \\ 1 & \text { for } m \leq 2 .\end{cases}
$$

The rigidity parameter serves as an input for the excluded volume expression and isotropic equation of state used in this work. It varies conveniently from zero to unity between the completely flexible- and rigid chain limit, respectively.

\section{THEORY}

Let us define the configuration $\tilde{\boldsymbol{r}}$ of a molecule by the position vector $\boldsymbol{r}$ of its center of mass together with the molecule's conformation $\tilde{\boldsymbol{\omega}}$. The latter is defined as the set of all bond- and torsion angles $\omega^{\prime}$ of the molecule and the orientation $\omega$ of the overall molecular axis with respect to some fixed reference frame. Analogous to our previous work, ${ }^{43}$ the molecular axis is defined as the axis around which the molecule has the smallest moment of inertia. The probability density to find any molecule in a configuration $\tilde{\boldsymbol{r}}$ is denoted by $\rho(\tilde{\boldsymbol{r}})$, which is normalized as

$$
\int \rho(\tilde{\boldsymbol{r}}) d \tilde{\boldsymbol{r}}=N .
$$

Here, $N$ is the number of molecules.

\section{A. Ideal part of the Helmholtz energy}

According to the general graphical and functional formalism developed by Chandler and Pratt, ${ }^{49,50}$ the ideal part of the Helmholtz energy of an inhomogeneous canonical system of $N$ nonrigid chain molecules can be written as a functional of $\rho(\tilde{\boldsymbol{r}})$ :

$$
\beta A^{\mathrm{id}}[\rho(\tilde{\boldsymbol{r}})]=\int_{V} \rho(\tilde{\boldsymbol{r}})\left[\ln \left(\rho(\tilde{\boldsymbol{r}}) \Lambda^{3}\right)-1+\beta \Phi_{\mathrm{intra}}(\tilde{\boldsymbol{r}})\right] d \tilde{\boldsymbol{r}} .
$$

Here, $\beta^{-1}$ is the product of the Boltzmann constant and the absolute temperature, $\Lambda$ is the thermal De Broglie wavelength, and $\Phi_{\text {intra }}(\tilde{\boldsymbol{r}})$ is the total intramolecular potential energy, containing all bonding and flexibility constraints. For the molecular model employed in this work, the bond length is fixed; therefore, the functional dependence of $\Phi_{\text {intra }}$ can be reduced to $\omega^{\prime}$. Furthermore, since we are concerned with describing nematic phases, the single-molecule density can be factorized into a uniform number density $\rho=N / V$ and a conformational distribution function $f(\tilde{\boldsymbol{\omega}})$ as $\rho(\tilde{\boldsymbol{r}})=\rho f(\tilde{\boldsymbol{\omega}})$. Consequently, all positional dependence of Eq. (3) can be averaged out and the ideal part of the Helmholtz energy becomes

$$
\begin{aligned}
\frac{\beta A^{\mathrm{id}}[f(\tilde{\boldsymbol{\omega}})]}{N}= & \ln \left(\rho \Lambda^{3}\right)-1+\int f(\tilde{\boldsymbol{\omega}})[\ln (f(\tilde{\boldsymbol{\omega}})) \\
& \left.+\beta \Phi_{\mathrm{intra}}\left(\boldsymbol{\omega}^{\prime}\right)\right] d \tilde{\boldsymbol{\omega}} .
\end{aligned}
$$


Since the conformational distribution function of a chain molecule in a condensed fluid is in general unknown, some approximations are required to transform this result into a practical functional for nematic fluids. In this, we follow the approach of Fynewever and Yethiraj ${ }^{23}$ and assume a molecule's internal configurational- $\left(\boldsymbol{\omega}^{\prime}\right)$ and orientational $(\boldsymbol{\omega})$ degrees of freedom can be decoupled

$$
f(\tilde{\boldsymbol{\omega}}) \approx f\left(\boldsymbol{\omega}^{\prime}\right) f(\boldsymbol{\omega}) .
$$

We note that this assumption is not completely in accordance with the increase in the end-to-end length of a molecule that is generally observed in molecular simulation studies of the isotropic-nematic phase transition. ${ }^{25,27,51}$ However, it is analogous to that underlying many accurate theories for isotropic fluids (e.g., SAFT, ${ }^{52}$ PC-SAFT ${ }^{53}$ ) where a similar increase in the end-to-end length for vapor-liquid phase transitions ${ }^{54}$ is neglected. Substituting Eq. (5) into Eq. (4), all $\boldsymbol{\omega}^{\prime}$-dependence can be averaged out to two temperature dependent terms. By generalizing the definition of the De Broglie wavelength (denoted by $\Lambda^{\prime}$ ), these temperature dependent terms can be considered in the isotropic part of the ideal Helmholtz energy. Accordingly, the ideal Helmholtz energy contribution of a nematic fluid of nonrigid tangent hard-sphere chain molecules can be put in a simple Onsager-like form, as

$$
\frac{\beta A^{\mathrm{id}}[f(\boldsymbol{\omega})]}{N}=\ln \left(\frac{\rho \Lambda^{\prime 3}}{\Omega}\right)-1+\int f(\boldsymbol{\omega}) \ln (\Omega f(\boldsymbol{\omega})) d \boldsymbol{\omega} .
$$

Here, $\Omega=\int d \omega=4 \pi$ is a normalization constant. The isotropic- and anisotropic contribution to the ideal Helmholtz energy are given by, respectively

$$
\begin{gathered}
\frac{\beta A_{\text {iso }}^{\mathrm{id}}}{N}=\ln \left(\frac{\rho \Lambda^{\prime 3}}{\Omega}\right)-1, \\
\frac{\beta A_{\text {aniso }}^{\mathrm{id}}[f(\boldsymbol{\omega})]}{N}=\int f(\boldsymbol{\omega}) \ln (\Omega f(\boldsymbol{\omega})) d \boldsymbol{\omega} .
\end{gathered}
$$

For an isotropic distribution of molecular orientations, $f_{\text {iso }}(\boldsymbol{\omega})=1 / \Omega$ and thus the anisotropic part is zero. For partial orientational order, the anisotropic contribution becomes larger than zero, corresponding to a decrease in orientational entropy.

\section{B. Residual part of the Helmholtz energy}

To describe the residual Helmholtz energy, let us write a virial expansion in the homogeneous number density $\rho$

$$
\frac{A^{\mathrm{res}}[f(\tilde{\boldsymbol{\omega}})]}{N k T}=B_{2}[f(\tilde{\boldsymbol{\omega}})] \rho+\frac{1}{2} B_{3}[f(\tilde{\boldsymbol{\omega}})] \rho^{2}+\ldots
$$

For molecules interacting through the pair-potential $\phi\left(\tilde{\boldsymbol{r}}_{1}, \tilde{\boldsymbol{r}}_{2}\right)$, the virial coefficients can be expressed as weighted integrals of the Mayer function $\left.\Phi\left(\tilde{\boldsymbol{r}}_{1}, \tilde{\boldsymbol{r}}_{2}\right)=\exp \left[-\beta \phi\left(\tilde{\boldsymbol{r}}_{1}, \tilde{\boldsymbol{r}}_{2}\right)\right)\right]-1$ over configurational space. ${ }^{55,56}$ The second virial coefficient, for example, can be written as

$$
B_{2}[f(\tilde{\boldsymbol{\omega}})]=-\frac{1}{2 V} \iint \Phi\left(\tilde{\boldsymbol{r}}_{1}, \tilde{\boldsymbol{r}}_{2}\right) f\left(\tilde{\boldsymbol{\omega}}_{1}\right) f\left(\tilde{\boldsymbol{\omega}}_{2}\right) d \tilde{\boldsymbol{r}}_{1} d \tilde{\boldsymbol{r}}_{2},
$$

where, for the purely repulsive molecules considered in this work, the integration over the positions results in a conformational average of the pair-excluded volume, according to

$$
B_{2}[f(\tilde{\boldsymbol{\omega}})]=\frac{1}{2} \iint V_{\mathrm{ex}}\left(\tilde{\boldsymbol{\omega}}_{1}, \tilde{\boldsymbol{\omega}}_{2}\right) f\left(\tilde{\boldsymbol{\omega}}_{1}\right) f\left(\tilde{\boldsymbol{\omega}}_{2}\right) d \tilde{\boldsymbol{\omega}}_{1} d \tilde{\boldsymbol{\omega}}_{2},
$$

$$
V_{\mathrm{ex}}\left(\tilde{\boldsymbol{\omega}}_{1}, \tilde{\boldsymbol{\omega}}_{2}\right)=-\int \Phi\left(\tilde{\boldsymbol{r}}_{1}, \tilde{\boldsymbol{r}}_{2}\right) d \boldsymbol{r}_{12} .
$$

Here, $\boldsymbol{r}_{12}$ is the vector between the center of mass of molecules 1 and 2. Since the conformational dependence of the pair-excluded volume is generally not known, we decouple the conformational distribution function as in Eq. (5) and treat the pair-excluded volume on an ensemble-average basis

$$
B_{2}\left[f(\boldsymbol{\omega}]=\frac{1}{2} \iint\left\langle V_{\mathrm{ex}}\left(\tilde{\boldsymbol{\omega}}_{1}, \tilde{\boldsymbol{\omega}}_{2}\right)\right\rangle_{\boldsymbol{\omega}_{1}^{\prime}, \boldsymbol{\omega}_{2}^{\prime}} f\left(\boldsymbol{\omega}_{1}\right) f\left(\boldsymbol{\omega}_{2}\right) d \boldsymbol{\omega}_{1} d \boldsymbol{\omega}_{2} .\right.
$$

The resulting ensemble average $\langle\ldots\rangle$ can be readily obtained from MC simulations of two chain molecules. ${ }^{23,43}$ Thereby, due to the averaging, it is a function solely of the angle $\gamma$ between the molecular axes, as

$$
\left\langle V_{\mathrm{ex}}\left(\tilde{\boldsymbol{\omega}}_{1}, \tilde{\boldsymbol{\omega}}_{2}\right)\right\rangle_{\boldsymbol{\omega}_{1}^{\prime}, \boldsymbol{\omega}_{2}^{\prime}}=V_{\mathrm{ex}}(\gamma) .
$$

In our previous work, ${ }^{43}$ the orientation-dependent excluded volume $V_{\mathrm{ex}}(\gamma)$ was calculated for a wide range of different rod-coil molecules. An analytical approximation was developed by correlating the $\mathrm{MC}$ simulation results:

$$
\begin{aligned}
V_{\mathrm{ex}}^{*}(\gamma) & =\frac{V_{\mathrm{ex}}(\gamma)}{V_{m}} \\
& =C_{1}\left(m, \chi_{\mathrm{R}}\right)+C_{2}\left(m, \chi_{\mathrm{R}}\right) \sin (\gamma)+C_{3}\left(m, \chi_{\mathrm{R}}\right) \sin ^{2}(\gamma) .
\end{aligned}
$$

Here, $V_{m}=(\pi / 6) m \sigma^{3}$ is the molecular volume of a chain of $m$ tangent hard spheres of diameter $\sigma$. The functional form of the coefficients $C_{1}, C_{2}$, and $C_{3}$, is listed in Appendix A. The correlation accurately covers the entire range of the rigidity parameter $\chi_{R}=[0,1]$ for chain lengths up to 30 . For longer chains, a small, systematic overestimation of the pairexcluded volume of more flexible chains is observed. ${ }^{43}$ It can be expected, however, that these small inaccuracies have a negligible effect on the prediction of the isotropic-nematic phase equilibrium. The reason is that, for the second-virial theories used in this work (discussed later in this section), the driving force, and thus the location (pressure/coexistence densities) of the isotropic-nematic phase transition is determined solely by the difference of the orientational average of the pair-excluded volume between an isotropic and nematic distribution of molecular orientations. This difference will be relatively unaffected by these errors. For linear chains, the correlation of Eq. (15) reduces to the quasi-exact result of Williamson and Jackson. ${ }^{57}$

To describe the higher order terms in the virial expansion from Eq. (9), two different approximate methods are evaluated in this work: the Vega-Lago rescaling, ${ }^{32}$ and Scaled Particle Theory (SPT). ${ }^{33-39}$ The approach of Vega and Lago is based on a simple rescaling of the higher virial coefficients to 
those of the isotropic fluid of the system being described, with

$$
B_{n}[f(\tilde{\boldsymbol{\omega}})] \approx B_{n, \text { iso }} \frac{B_{2}[f(\tilde{\boldsymbol{\omega}})]}{B_{2, \text { iso }}} .
$$

Although the rescaling of virial coefficients might seem $a d$ hoc in nature, it has a sound statistical mechanical basis in the form of the decoupling approximation, ${ }^{19,29-31}$ which is briefly discussed in Appendix B. Resuming the virial expansion from Eq. (9), and using Eq. (13) for the second virial coefficient, leads to the residual Helmholtz energy, according to

$$
\frac{\beta A^{\text {res }}[f(\boldsymbol{\omega})]}{N}=\frac{\beta A_{\text {iso }}^{\text {res }}}{N} \frac{B_{2}[f(\boldsymbol{\omega})]}{B_{2, \text { iso }}} .
$$

Upon differentiation, the compressibility factor $Z \equiv P V$ $/ N k T$ is obtained as

$$
Z=1+Z_{\text {iso }}^{\text {res }} \frac{B_{2}[f(\omega)]}{B_{2, \text { iso }}} .
$$

Clearly, a major advantage of using the rescaling is that the conformation dependence of the complete virial expansion is approximately considered in the second virial coefficient. Due to the use of Eqs. (13)-(15) for the second virial coefficient, the resulting expressions retain a functional dependence on the orientational distribution function only. An Onsagerlike theory for non-rigid chain molecules is thereby obtained. Furthermore, the rescaling allows for the use of an accurate EoS for the description of the isotropic fluid. In this work, we evaluate the Liu-Hu (LH) $\mathrm{EoS}^{47}$ and an extension of the LH EoS to partially flexible (rod-coil) and linear hard-sphere chain fluids for this. ${ }^{48}$ In the remainder of this paper, this extended EoS will be referred to as the LHrc EoS (where rc is an abbreviation for "rod-coil"). In a previous work ${ }^{48}$ we have shown that, compared to the LH EoS, the LHrc EoS leads to a more reliable description of the isotropic equation of state of linear and partially flexible (rod-coil) tangent hard-sphere chain fluids. It is interesting to evaluate to what extend these improved results for the isotropic fluid are reflected in the predicted isotropic-nematic phase equilibrium. The second virial coefficient of the isotropic fluid that is required in Eqs. (17) and (18) can be obtained by substituting $f_{\text {iso }}(\boldsymbol{\omega})=1 / 4 \pi$ in Eqs. (13)-(15):

$$
B_{2, \text { iso }}=V_{\mathrm{m}}\left[\frac{C_{1}\left(m, \chi_{\mathrm{R}}\right)}{2}+\pi \frac{C_{2}\left(m, \chi_{\mathrm{R}}\right)}{8}+\frac{C_{3}\left(m, \chi_{\mathrm{R}}\right)}{3}\right] .
$$

In the remainder of this paper, Eqs. (6), (13)-(15), and (17)-(19) will be referred to as the Onsager Vega-Lago (OVL) theory.

In contrast to the OVL theory, SPT cannot be derived directly from the virial expansion. However, SPT shows the same features in that the higher virial terms are approximated by a nonlinear dependence on density whereas the second virial coefficient is treated explicitly. By combining the work of Cotter ${ }^{33-35}$ and Boublík et al.,${ }^{36-39}$ Jaffer et al. ${ }^{58}$ proposed a SPT that is directly applicable to describe both the isotropic and nematic phase of tangent hard-sphere chain fluids. The residual Helmholtz energy and compressibility factor obtained from this SPT are given by, respectively

$$
\begin{aligned}
& \frac{\beta A^{\text {res }}}{N}=(\psi-1) \ln (1-\eta)+\frac{3 a \eta}{1-\eta}+\frac{\psi \eta}{(1-\eta)^{2}}, \\
& Z=\frac{1}{1-\eta}+\frac{3 a \eta}{(1-\eta)^{2}}+\frac{3 \psi \eta^{2}}{(1-\eta)^{3}}-\frac{\psi \eta^{3}}{(1-\eta)^{3}} .
\end{aligned}
$$

Here, $\eta=\rho V_{\mathrm{m}}$ is the packing fraction of the system. Using the definition of the reduced second virial coefficient $B_{2}^{*}=B_{2} / V_{m}=\partial Z /\left.\partial \eta\right|_{\eta=0}$, the non-sphericity parameter $a$ can be written as

$$
a=\frac{B_{2}^{*}-1}{3} .
$$

Since the non-sphericity parameter is expressed explicitly in the reduced second virial coefficient, the SPT can be directly applied to nematic fluids through the use of Eqs. (13)-(15). Please note that in related work, the non-sphericity parameter is usually referred to as $\alpha$. Since this symbol will be used as the variational parameter for the Onsager Trial function (see Sec. III C) we have used the symbol $a$ instead. The reduced second virial coefficient in the isotropic phase can be calculated from Eq. (19). The $\psi$-parameter in Eq. (20) is given by ${ }^{58}$

$$
\psi=\frac{\sigma S_{m}}{9 V_{m}}\left[3 a-\left(\frac{\sigma S_{m}}{4 V_{m}}\right)\left(1-\frac{m-1}{4}\right)\right] .
$$

Here, $S_{m}=\pi m \sigma^{2}$ is the surface area of a chain molecule. Equations (6), (13)-(15), and (19)-(23) will be referred to as SPT.

\section{Solving the phase equilibrium}

In principle, phase equilibrium is attained when two phases are in thermal, mechanical, and material equilibrium. The conditions for this are equality of temperature $(T)$, pressure $(P)$, and chemical potential $(\mu)$, respectively. For the hard molecules considered in this work, however, temperature is an irrelevant parameter. Consequently, for these systems the phase equilibrium can be solved by equating the pressure $P$ and chemical potential $\mu$ of both phases

$$
\begin{aligned}
& P^{\text {iso }}\left(\rho_{\text {iso }}\right)=P^{\text {nem }}\left[f_{\text {eq }}(\boldsymbol{\omega}) ; \rho_{\text {nem }}\right], \\
& \mu^{\text {iso }}\left(\rho_{\text {iso }}\right)=\mu^{\text {nem }}\left[f_{\text {eq }}(\boldsymbol{\omega}) ; \rho_{\text {nem }}\right] .
\end{aligned}
$$

The equilibrium orientational distribution function $f_{\mathrm{eq}}(\boldsymbol{\omega})$ minimizes the total Helmholtz energy $A=A^{\text {id }}+A^{\text {res }}$ and can be obtained by solving the following variational equation:

$$
\left(\frac{\delta A[f(\boldsymbol{\omega})]}{\delta f(\boldsymbol{\omega})}\right)_{N V T, f(\boldsymbol{\omega})=f_{\mathrm{eq}}(\boldsymbol{\omega})}=0 .
$$

The pressure and chemical potential can be obtained from

$$
\begin{gathered}
P=-\left(\frac{\partial A\left[f_{\mathrm{eq}}(\boldsymbol{\omega})\right]}{\partial V}\right)_{N T}, \\
\mu=\left(\frac{\partial A\left[f_{\mathrm{eq}}(\boldsymbol{\omega})\right]}{\partial N}\right)_{V T} .
\end{gathered}
$$


Although, in principle Eq. (26) can be solved by numerical techniques, ${ }^{59-64}$ a well chosen trial function can make the solution more tractable without losing too much of the numerical accuracy. ${ }^{12}$ In this work, we use the hyperbolic trial function as originally proposed by Onsager ${ }^{11}$ (referred to as OTF) and consider the degree of orientational order in a single parameter $\alpha$ :

$$
f(\boldsymbol{\omega}) \approx f_{\mathrm{OTF}}(\theta)=\frac{\alpha \cosh [\alpha \cos (\theta)]}{4 \pi \sinh (\alpha)} .
$$

Here, $\theta$ is the polar angle of a molecule's axis with respect to the nematic director (mean direction of all molecules). For an isotropic phase $f_{\mathrm{OTF}}=1 / 4 \pi$ and thus $\alpha=0$. For higher values of $\alpha$, the OTF becomes sharply peaked at the parallel orientations $\theta=0$ and $\theta=\pi$, thereby modeling a nematic phase. The use of the OTF allows the orientation-dependent terms of the Helmholtz energy (Eqs. (8) and (13)) to be expressed as a function solely of $\alpha$ (see Appendix $\mathrm{C}$ for details). Accordingly, the variational problem from Eq. (26) can be transformed to a simple one-dimensional parameter optimization of the equilibrium degree of orientational order $\alpha_{\mathrm{eq}}$, as

$$
\left(\frac{\partial A(\alpha)}{\partial \alpha}\right)_{N V T, \alpha=\alpha_{\mathrm{eq}}}=0
$$

A modified Newton method was used to solve this equation. The resulting $\alpha=\alpha_{\text {eq }}$ determines the orientational distribution function $f(\boldsymbol{\omega})$ according to Eq. (29). Therefore, the ne-

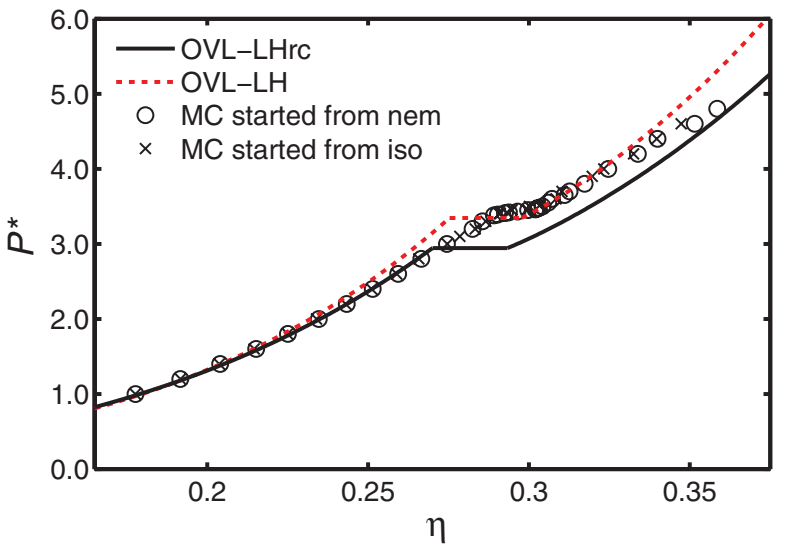

(a)

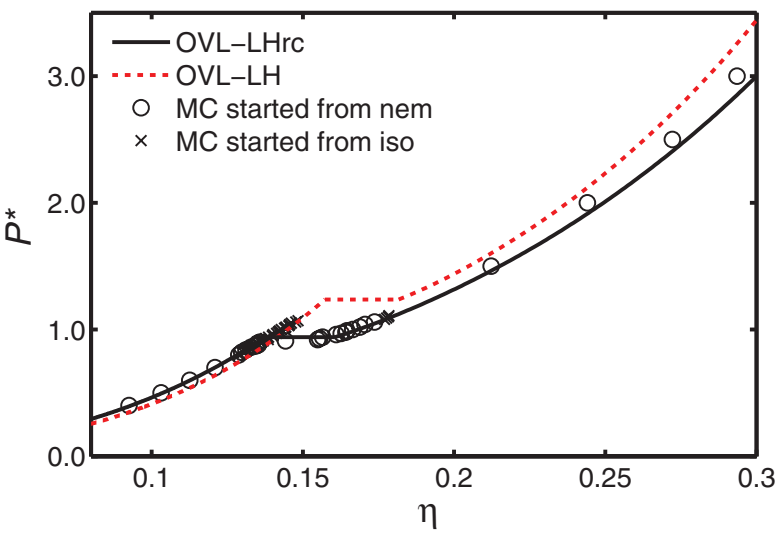

matic order parameter $S_{2}$ can be calculated as

$$
\begin{aligned}
S_{2} & =\left\langle\frac{3}{2} \cos ^{2}(\theta)-1 / 2\right\rangle_{\omega}, \\
& =1-\frac{3 \operatorname{coth}(\alpha)}{\alpha}+\frac{3}{\alpha^{2}} .
\end{aligned}
$$

The nematic order parameter varies between zero and unity for the isotropic and perfect nematic phase, respectively. It is therefore a convenient measure of the degree of orientational order in a system.

\section{RESULTS}

In this section, theoretical results for the pressure and nematic order parameter are compared to the results from isobaric-isothermal NPT MC simulations from Ref. 27. To show the effect of hysteresis around the phase transition, both the results from $\mathrm{MC}$ simulations started from an isotropic and a nematic initial configuration are included.

\section{A. Linear $m$-mers}

First we assess the accuracy of the OVL theory in predicting the isotropic-nematic phase equilibrium of linear chains. In Fig. 1, the equation of state of a system of linear 7-, 11-, 15-, and 20-mers as obtained from the OVL-LH and OVLLHrc theory is compared to $\mathrm{MC}$ simulations. The results show
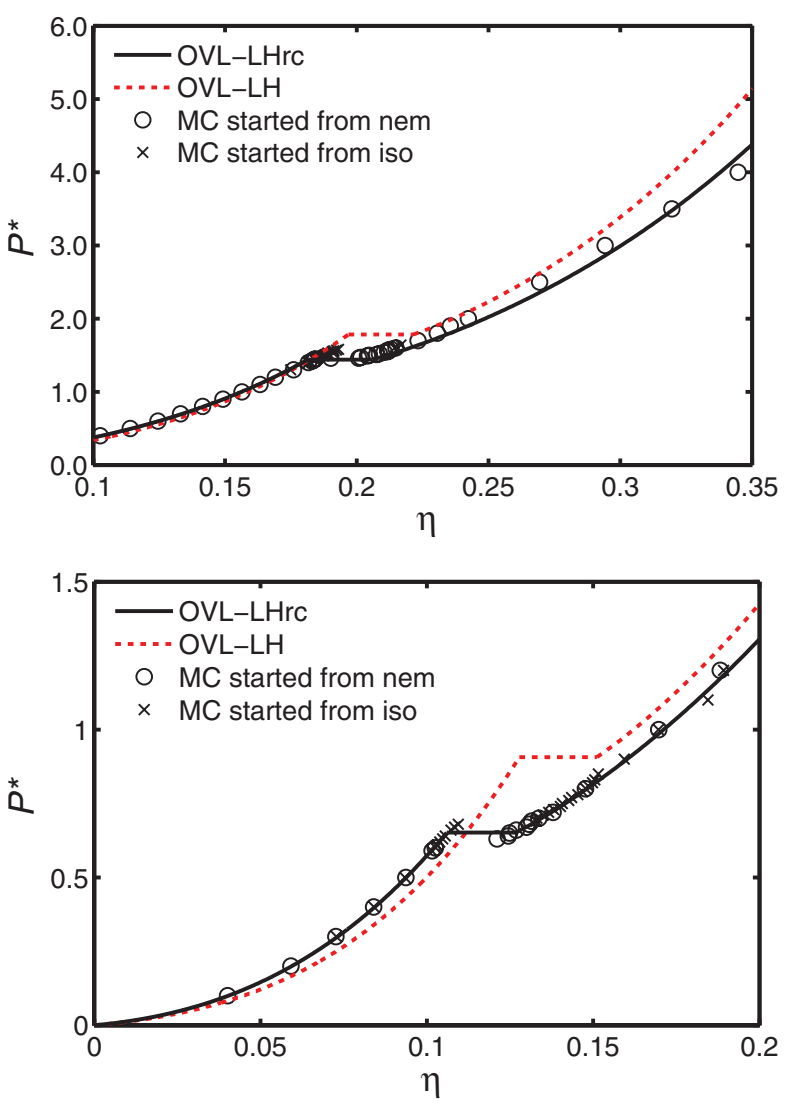

FIG. 1. The equation of state of a system of (a) linear 7-mers, (b) linear 11-mers, (c) linear 15-mers, and (d) linear 20-mers, as predicted from the OVL theory using the LHrc (solid line) and LH (dotted line) equations of state as input compared to MC simulations ${ }^{27}$ (symbols). The MC simulations were started either from an isotropic or a nematic initial configuration. 
a large sensitivity of the OVL theory towards the EoS that is used for the isotropic phase. It is rewarding to see that the relatively small improvement in the description of the isotropic phase obtained from using the LHrc EoS results in a considerably more accurate description of the isotropic-nematic phase equilibrium. The deviation of the theory from simulations observed for the 7-mer system, points at limitations in the use of the rescaling of virial coefficients from Eq. (16). For smaller chain lengths, the phase equilibrium is shifted to higher packing fractions; therefore, more error is introduced due to the approximate treatment of the higher virial coefficients in Eq. (16). The observation that, for this system, the OVL-LH theory results in a better prediction of the phase transition pressure and nematic branch, is most likely caused by a fortuitous cancellation of errors from the approximate rescaling of virial coefficients and the inaccurate description of the isotropic equation of state in Eq. (18). In fact, given that the chemical potentials of both phases are equal at the phase equilibrium, we can write for the phase transition pressure

$$
P_{\text {trans }}=-\frac{a_{\text {nem }}-a_{\text {iso }}}{v_{\text {nem }}-v_{\text {iso }}} .
$$

Here, $a$ and $v$ are the molar Helmholtz energy and volume, respectively. Accordingly, the observation that $P_{\text {trans }}$ is predicted correctly while the difference in coexistence packing fractions (and thus molar volumes) is overestimated, can only be due to an overestimation of the isotropic-nematic Helmholtz energy difference. Consistently, the nematic order parameter obtained from the OVL-LH theory for this system (see Fig. 2(a)) is also overestimated.

In Fig. 2, the nematic order parameter obtained from the OVL-LHrc theory is compared to that obtained from the OVL-LH theory and MC simulations for the same systems as in Fig. 1. For the OVL-LHrc theory, the results show consistency with those obtained for the equation of state in Fig. 1. For the linear 11-, 15-, and 20-mer systems, the theory is in excellent agreement with the MC data. As indicated by the results from Fig. 3, where we compare predictions based on the OTF and a full numerical solution of the orientational distribution function, the small overestimation of the order parameter close to the phase equilibrium is most probably an artifact of the use of the OTF. For the OVL-LH theory, the seemingly accurate comparison with $\mathrm{MC}$ data for the order parameter of the linear 11-mer system (Fig. 2(b)) is merely an effect of the overestimation of the nematic coexistence packing fraction.

In Figs. 4 and 5, we show the results obtained from SPT (dotted lines) for the same systems as in Figs. 1 and 2. The results obtained from the OVL-LHrc theory (solid lines) are also included for comparison. The predicted equation of state and nematic order parameter obtained from both theories are very similar. Quantitative agreement between theory and MC data is obtained for the linear 11-, 15-, and 20-mer systems.
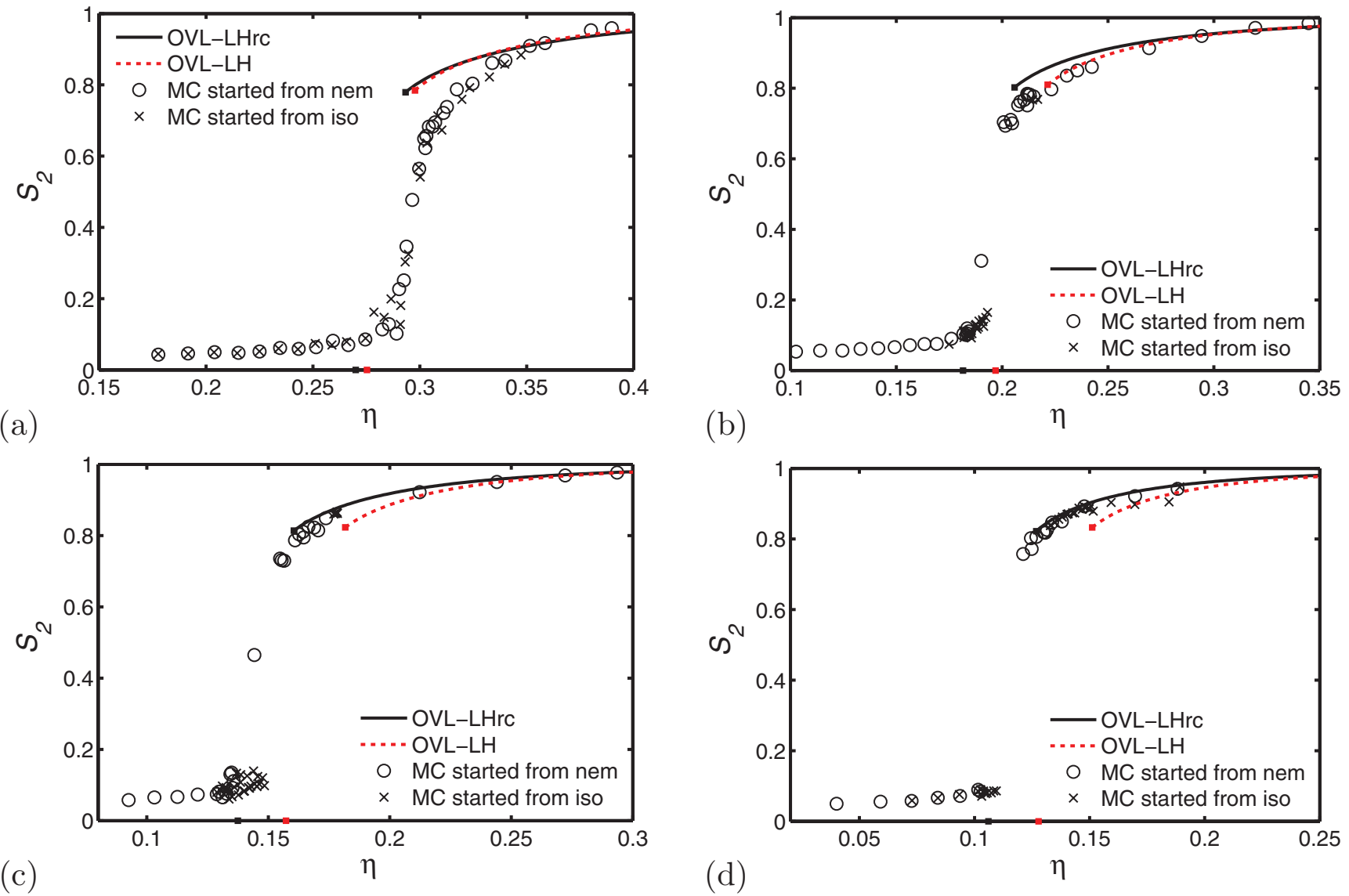

(b)

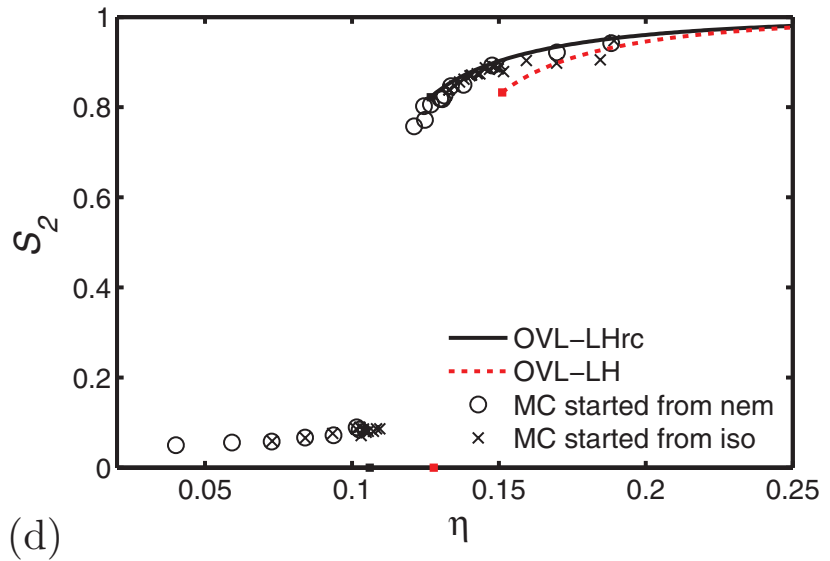

FIG. 2. The nematic order parameter $S_{2}$ of a system of (a) linear 7-mers, (b) linear 11-mers, (c) linear 15-mers, and (d) linear 20-mers, as predicted from the OVL theory using the LHrc (solid line) and LH (dotted line) equations of state as input compared to MC simulations ${ }^{27}$ (symbols). The MC simulations were started either from an isotropic or a nematic initial configuration. 
(a)

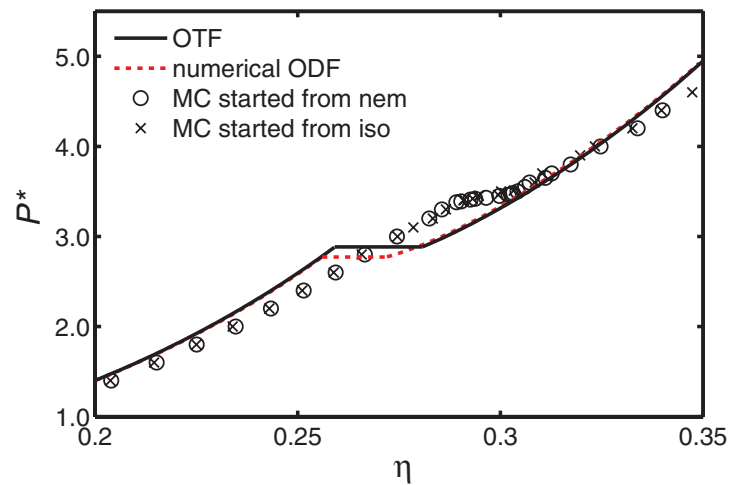

(b)

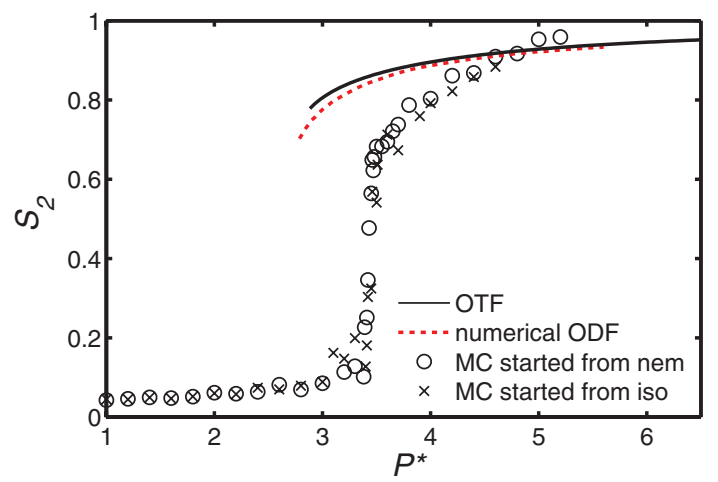

FIG. 3. The equation of state and nematic order parameter of a system of linear 7-mers as obtained from OVL-TPT1 based on the OTF (solid line) and a full numerical solution of the orientational distribution function ${ }^{24}$ (dotted line) compared to MC simulations ${ }^{27}$ (symbols). The MC simulations were started either from an isotropic or a nematic initial configuration.

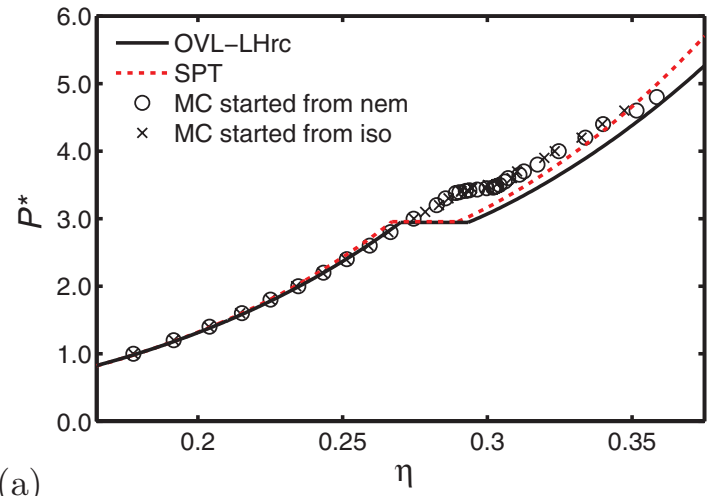

(a)

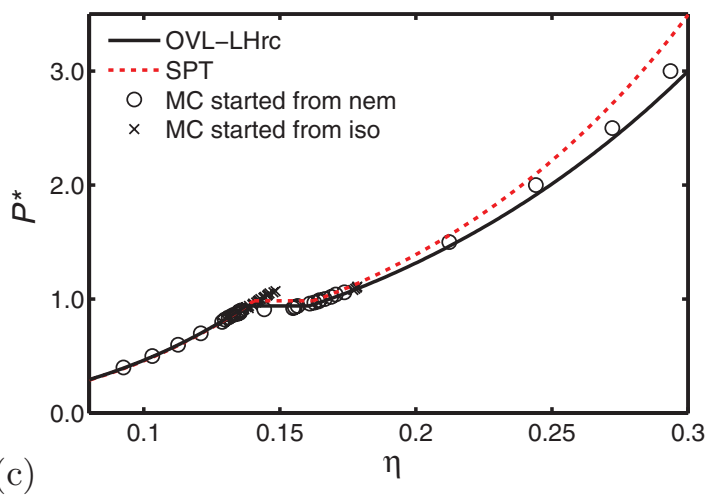

As indicated by the results for the linear 7-mer system, SPT suffers from the same shortcomings as the OVL theory and results in a less accurate description of the phase transition for smaller chain lengths. A comparison of results obtained from SPT and the OVL-LHrc theory to the MC simulations from Ref. 27 (not included for brevity) showed that both theories are accurate down to a chain length of 9 , resulting in an underestimation of the coexistence pressure, isotropic packing fraction, and nematic packing fraction of less than 7\%, 5\%, and $1 \%$, respectively.

In Fig. 6, the density difference $\Delta \eta$ at the phase transition as obtained from the OVL-LHrc theory, SPT, and MC simulations is plotted against the chain length $m$. As is common with theories of this type, both OVL-LHrc and SPT result in an overestimation of the density difference for small chain lengths. For larger chain lengths, however, theoretical results are in excellent agreement with the MC simulations. Although the MC data only include chain lengths up to 20, the observation that the theory becomes more accurate for longer chain lengths suggests these results can be extrapolated to larger chain lengths as well. For very large chain lengths, the coexistence densities obtained from SPT and OVL-LHrc converge to the same constant values (corresponding to the Onsager limit) and a constant density difference (relative to the isotropic coexistence density) of approximately $34 \%$ is obtained.

It is important to note that in molecular simulation studies of linear tangent hard-sphere chain fluids, ${ }^{65}$ no nematic phase is observed for chain lengths smaller than 5 . The reason is that for such short chains the isotropic-nematic phase

(b)
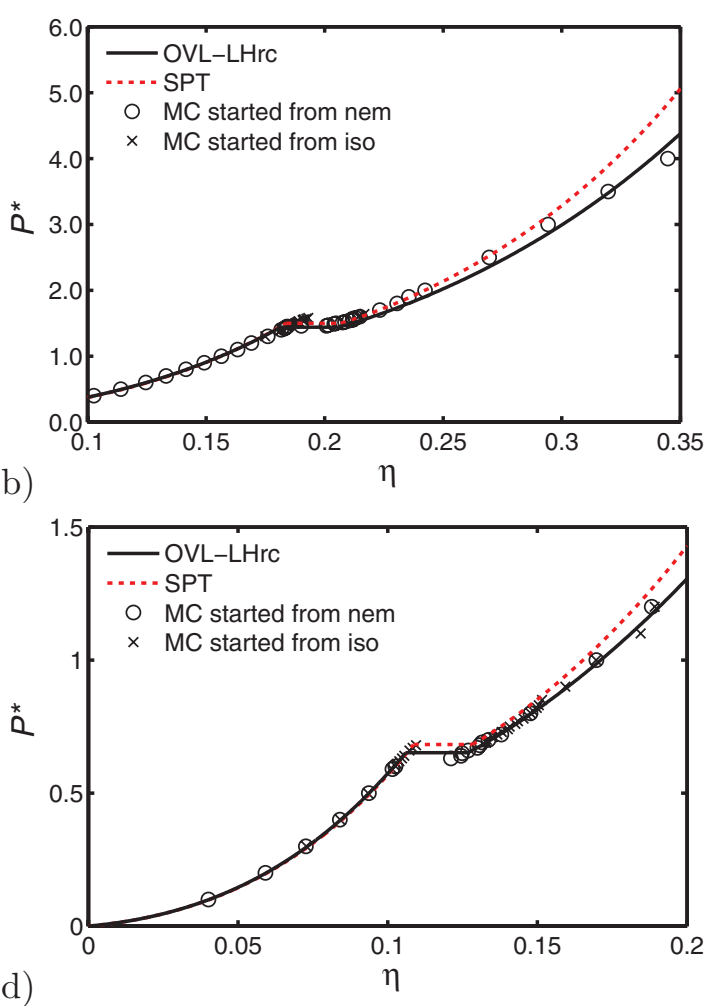

FIG. 4. The equation of state of a system of (a) linear 7-mers, (b) linear 11-mers, (c) linear 15-mers, and (d) linear 20-mers, as predicted from OVL-LHrc (solid line) and SPT (dotted line) compared to MC simulations ${ }^{27}$ (symbols). The MC simulations were started either from an isotropic or a nematic initial configuration. 


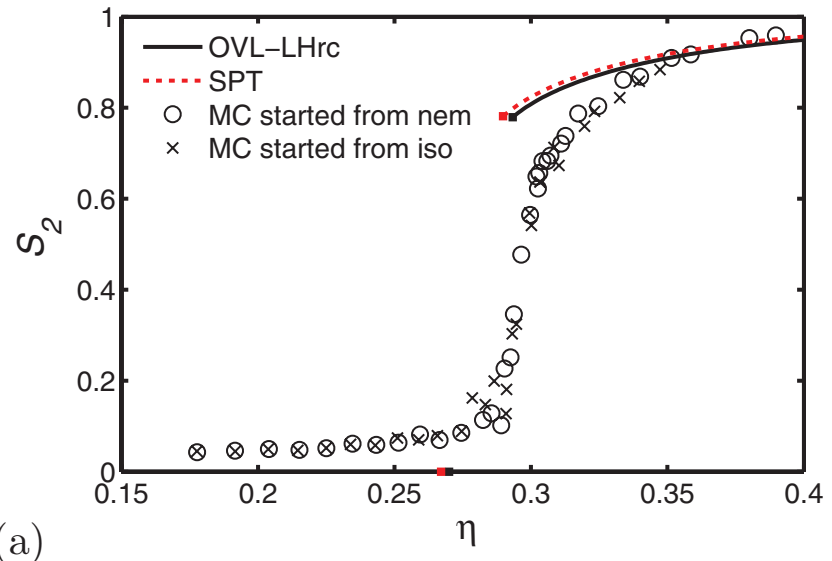

(a)

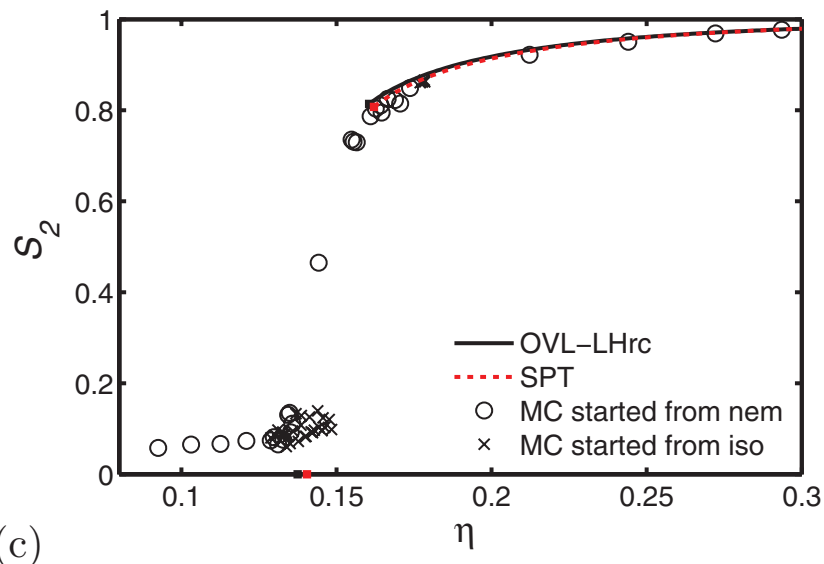

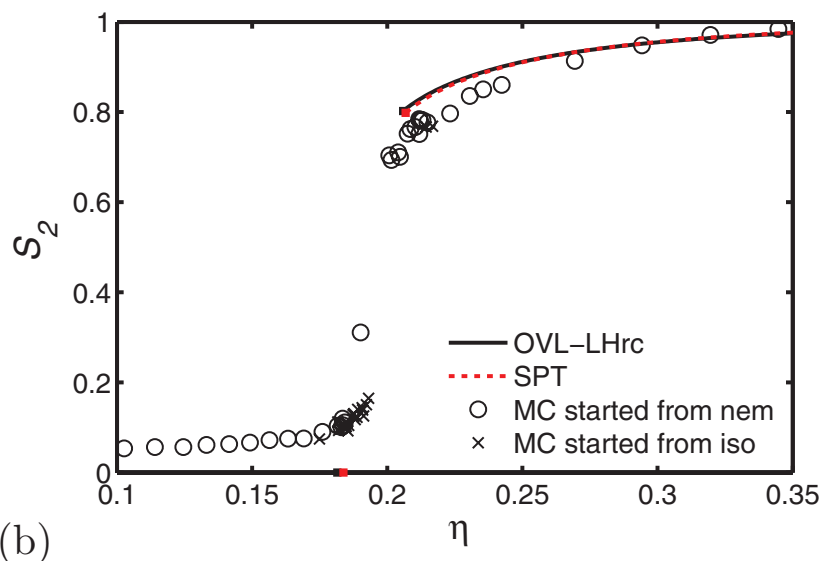

(b)

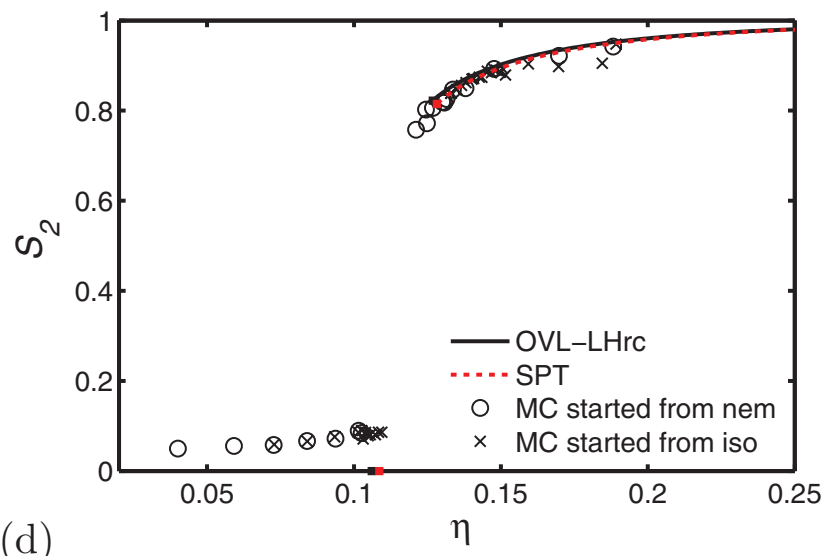

FIG. 5. The nematic order parameter $S_{2}$ of a system of (a) linear 7-mers, (b) linear 11-mers, (c) linear 15-mers, and (d) linear 20-mers, as predicted from OVL-LHrc (solid line) and SPT (dotted line) compared to MC simulations ${ }^{27}$ (symbols). The MC simulations were started either from an isotropic or a nematic initial configuration.

equilibrium is shifted to packing fractions beyond the isotropic-solid phase equilibrium. The isotropic-nematic coexistence packing fractions obtained from the theory are in accordance with these results. For example, for a linear 3- and 4-mer, the OVL-LHrc theory predicts isotropic-nematic coexistence packing fractions of $\eta_{\text {iso }}=0.541$ and $\eta_{\text {nem }}=0.551$, and $\eta_{\text {iso }}=0.433$ and $\eta_{\text {nem }}=0.448$, respectively. These values

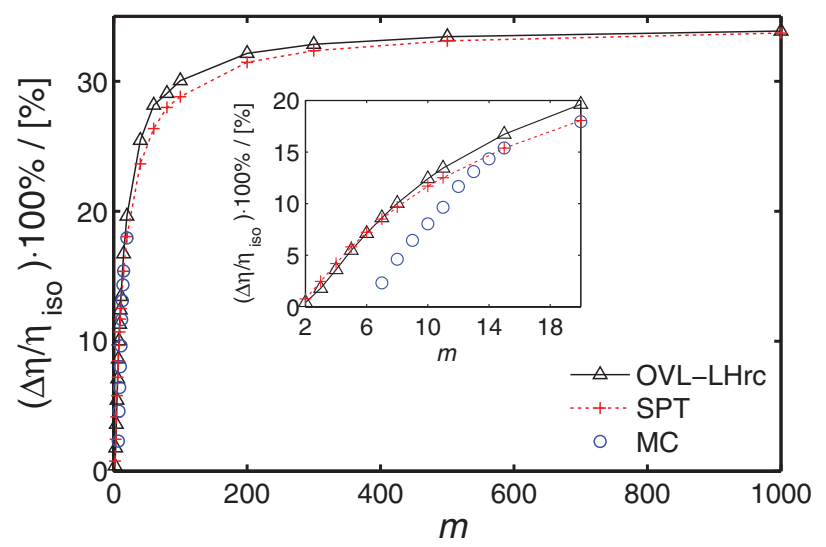

FIG. 6. The chain-length-dependence of the density difference $\Delta \eta$ at the isotropic-nematic phase transition. Comparison between predictions obtained from OVL-LHrc (triangles), SPT (plus signs), and MC simulations ${ }^{27}$ (circles). The lines are a guide for the eye. The solid line corresponds to OVLLHrc; the dotted line corresponds to SPT. are larger than the isotropic-solid coexistence packing fractions obtained from molecular simulations, ${ }^{65}$ i.e., $\eta_{\text {iso }} \geq 0.430$ and $\eta_{\text {solid }} \leq 0.529$ for a linear 3 -mer, and $\eta_{\text {iso }}=0.43$ and $\eta_{\text {solid }}$ $=0.51$ for a linear 4-mer. Since the theory presented in this work does not include a description of the solid phase, no direct comparison between theory and molecular simulations can be made for the isotropic-solid coexistence packing fractions.

\section{B. Rod-coil fluids}

Given the results obtained in Sec. IV A for linear $m$-mers, only SPT and the OVL-LHrc theory are evaluated further for the description of rod-coil fluids. The equation of state and nematic order parameter obtained from these theories are compared to MC simulations in Figs. 7 and 8, respectively. The test systems included are (a) a linear 15-mer, (b) a 15-14 rodcoil, (c) a 15-13 rod-coil, and (d) a 15-12 rod-coil. For all systems shown, the overall agreement of SPT and the OVL-LHrc theory with MC simulations is satisfactory. The general trends with molecular rigidity are well captured. It can be clearly observed that for less rigid chains, the phase transition is shifted to higher packing fractions. The reason is that the shape of such molecules is less anisotropic. Accordingly, the difference of the orientational average of the pair-excluded volume between an isotropic and nematic distribution of molecular 


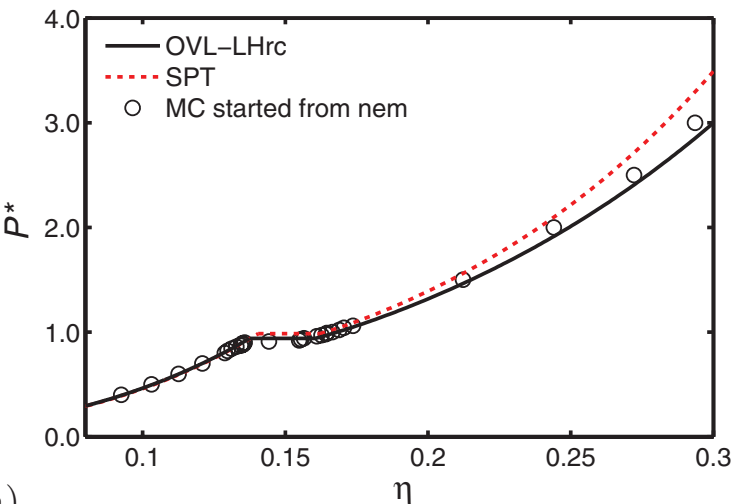

(b)

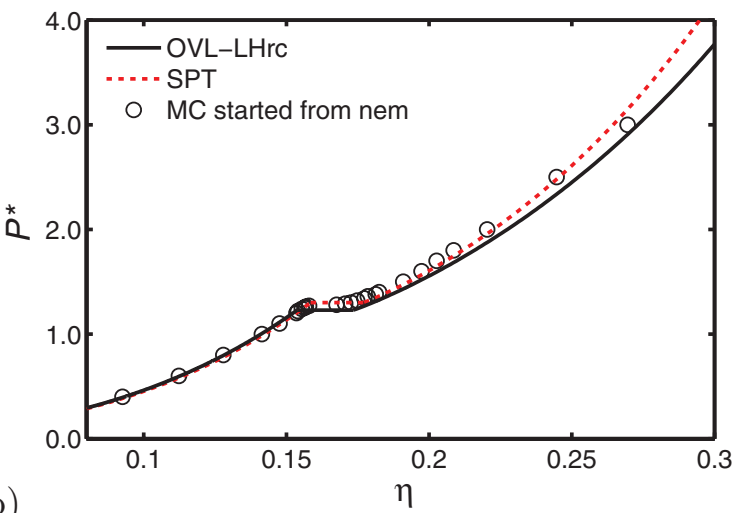

(a)

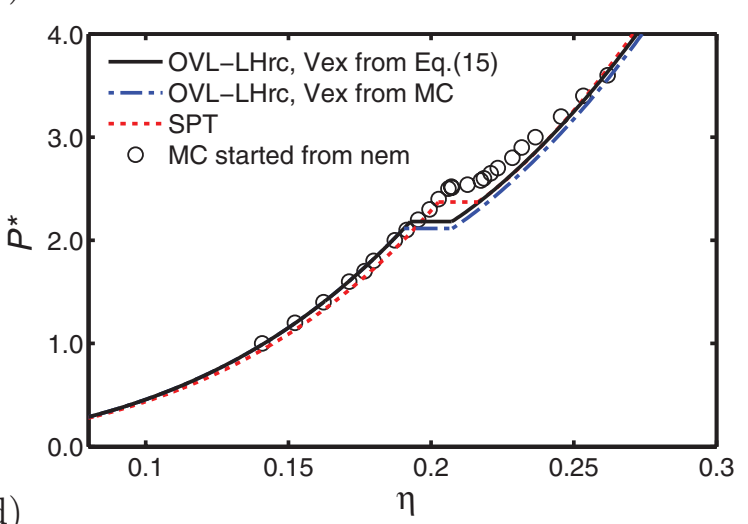

(c)

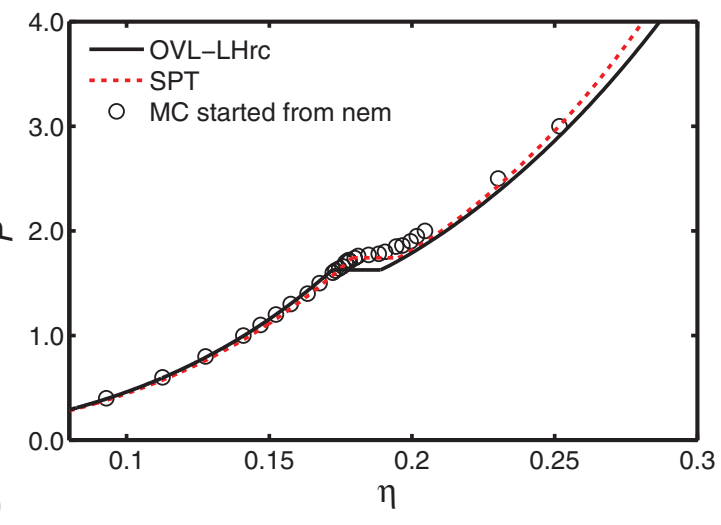

(d)

FIG. 7. The equation of state of a system of (a) linear 15-mers, (b) 15-14 rod-coils, (c) 15-13 rod-coils, and (d) 15-12 rod-coils, as predicted from OVL-LHrc (solid line) and SPT (dotted line) compared to MC simulations ${ }^{27}$ (symbols). To check the effect of using the correlation from Eq. (15) for the pair-excluded volume, the pair-excluded volume of the 15-12 rod-coil was calculated from MC simulations (using the method from Ref. 43). A third order series in sin ( $\gamma$ ) essentially gave a perfect fit. The results obtained from OVL-LHrc based on this fit are included (dash-dotted line) in (d). It is rewarding to see that both approaches practically yield the same results.
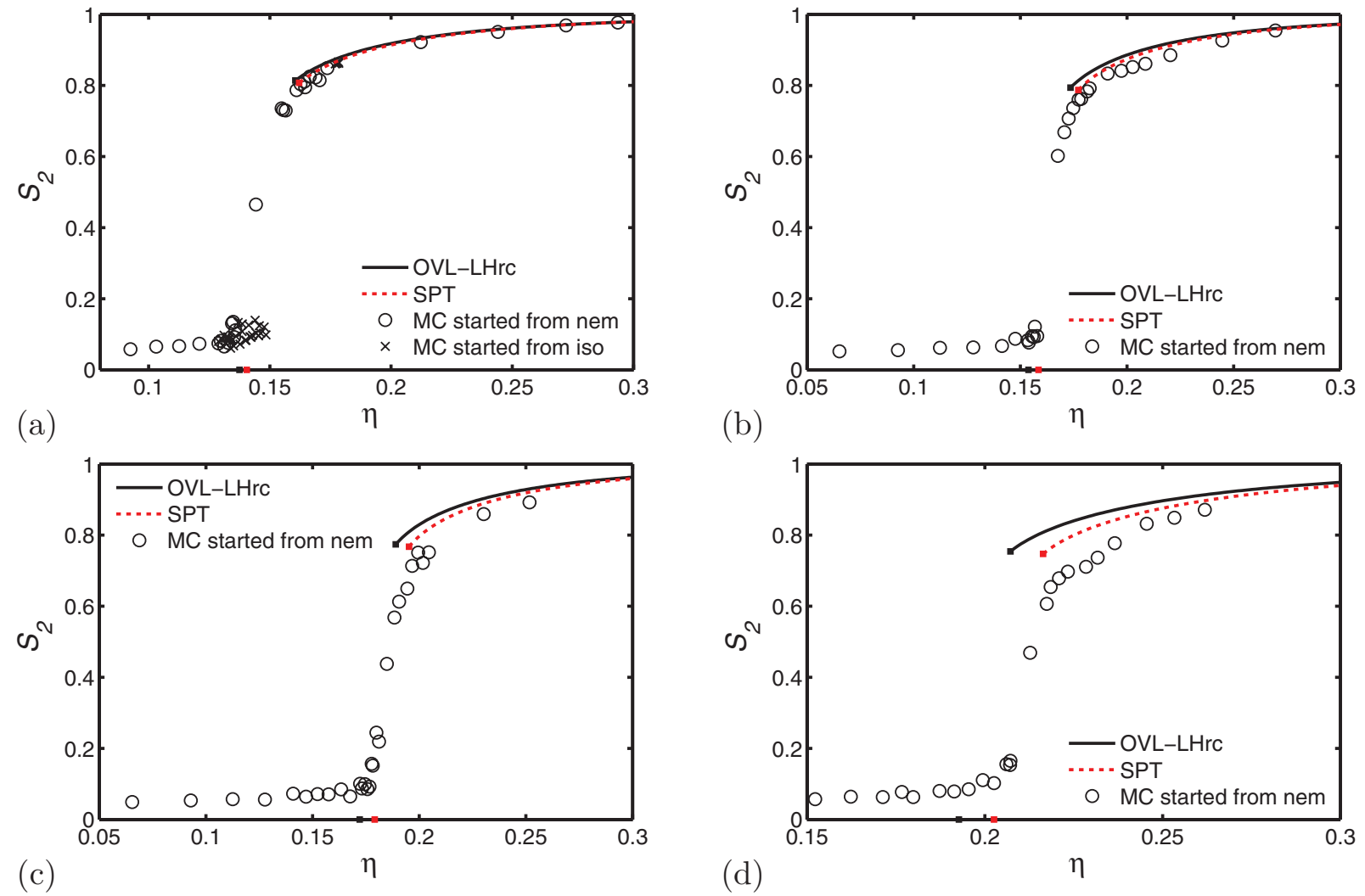

FIG. 8. The nematic order parameter $S_{2}$ of a system of (a) linear 15-mers, (b) 15-14 rod-coils, (c) 15-13 rod-coils, and (d) 15-12 rod-coils, as predicted from OVL-LHrc (solid line) and SPT (dotted line) compared to MC simulations ${ }^{27}$ (symbols). 
orientations (as obtained from Eq. (15)) is smaller, resulting in a smaller driving force for the phase transition.

As shown in Figs. 7 and 8, both SPT and the OVLLHrc theory results in a small underestimation of the location (pressure and coexistence densities) of the phase transition for more flexible chains. Part of the reason is that, due to the increased flexibility of the molecules, the phase transition is shifted to higher packing fraction and thus errors introduced by the approximate treatment of the higher virial coefficients become apparent. As shown previously for linear $m$-mers, inaccuracies of this kind result in an underestimation of the location of the phase transition. Compared to linear chains, however, the underestimation seems to be more severe. This could be due to several reasons. The first, and most probable, reason is that the approximate description of the higher virial coefficients becomes less accurate for chains of increased flexibility. Second, it could be due to the introduction of small inaccuracies through the use of the correlation for the pair-excluded volume from Eq. (15). To test this hypothesis, we calculated the orientation-dependent pair-excluded volume of a 15-12 rod-coil using the Monte Carlo method from Ref. 43. A third order series in $\sin (\gamma)$ (i.e., $V_{\text {ex }}(\gamma)=\sum_{i=1}^{4} C_{i} \sin ^{i-1}(\gamma)$, with $C_{1}=22.5871, C_{2}$ $=17.1903, C_{3}=26.4334$, and $\left.C_{4}=-16.3469\right)$ gave a nearly perfect fit. Calculations of the OVL-LHrc theory based on this fit are included (dash-dotted line) in Fig. 7(d). It is comforting to see that the difference with the results obtained from using Eq. (15) is very small. Moreover, the use of the fit leads to a slightly lower - not higher - prediction of the location of phase transition. Finally, the reason could be the decoupling of the conformational distribution function in Eq. (5). First of all, the decoupling is in contradiction to the increase in end-toend length at the isotropic-nematic phase transition. Given the results from Ref. 27 (i.e., increase end-to-end-length $<2 \%$ ), however, it is unlikely this effect is significant here. Furthermore, the increase in end-to-end length has two opposite effects on the driving force of the phase transition (a negative effect due to a decrease in chain-conformational entropy and a positive effect due to an increase in shape anisotropy of the molecules) which may just as well cancel each other out. The second implication of the decoupling is of more fundamental nature. Essentially, the decoupling comes down to modeling a (partially) flexible molecular model by a cylindrically symmetric, rigid molecular model the pair-excluded volume interaction of which can be described by Eq. (14). It could be that due to the averaging, part of the destabilizing effect of molecular flexibility on the isotropic-nematic phase transition is lost. Having said that, it seems reasonable to assume these effects are minor for the relatively stiff chains shown in Figs. 7 and 8. In conclusion, we expect the approximate treatment of the higher virial coefficients to cause the deviations of theory to simulations in Figs. 7 and 8.

In Fig. 9, we show the density difference $\Delta \eta$ at the phase transition and part of the phase diagram, respectively, of a $15-m_{\mathrm{R}}$ rod-coil. Compared to the MC data, ${ }^{27}$ both SPT and the OVL-LHrc theory capture the general trends with respect to the rigidity parameter, i.e., for more flexible chains the phase transition is shifted to higher packing fraction and the density difference (relative to the isotropic coexistence density) (a)

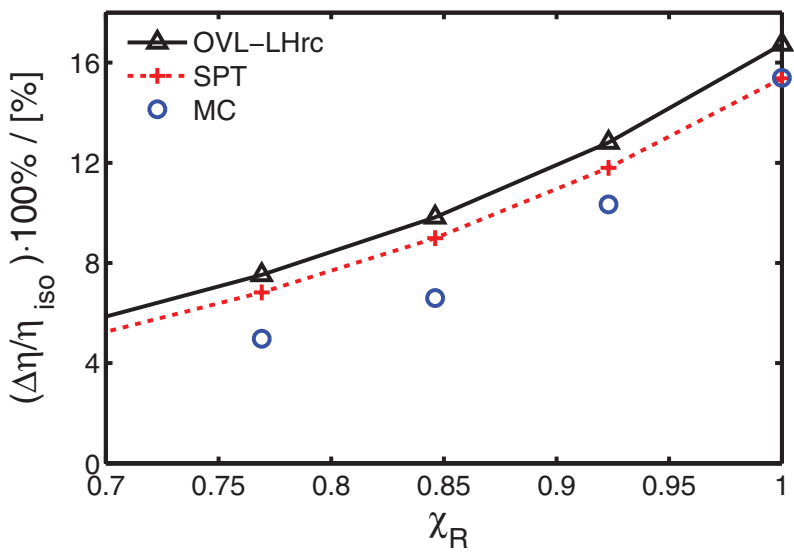

(b)

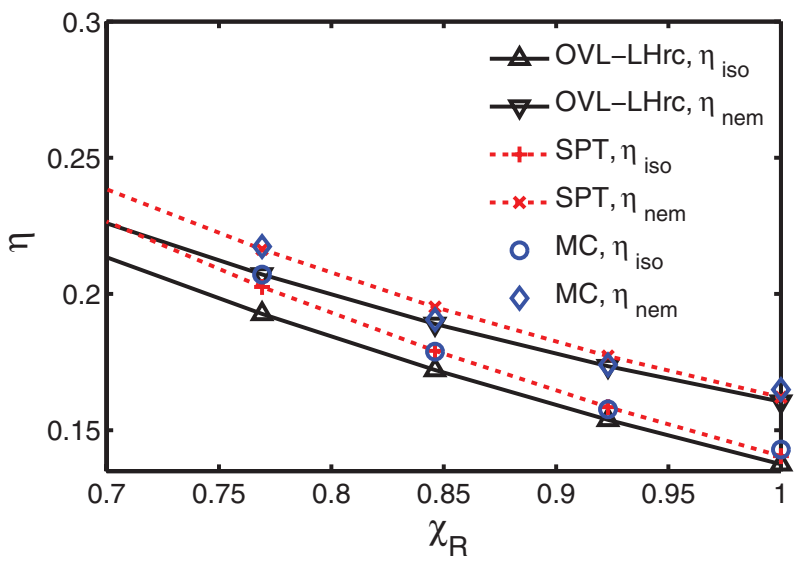

FIG. 9. The variation of (a) the density difference $\Delta \eta$ at the isotropicnematic phase transition and (b) the phase diagram with the rigidity parameter $\chi_{\mathrm{R}}$ for a $15-m_{\mathrm{R}}$ rod-coil. Comparison between predictions obtained from OVL-LHrc (solid line) and SPT (dotted line), compared to MC simulations ${ }^{27}$ (symbols). The lines are a guide for the eye. The solid line corresponds to OVL-LHrc; the dotted line corresponds to SPT.

is decreased. For all rod-coils shown, however, an overestimation of the density difference is observed. It is important to note that Jiang and $\mathrm{Wu}^{40}$ studied the same test system using SPT with a numerical solution of the ODF. Their results do not differ significantly from those shown in Fig. 9 - justifying the use of the OTF. Interestingly, Jiang and $\mathrm{Wu}$ found isotropic-nematic coexistence for relatively stiff chains only $\left(\chi_{\mathrm{R}} \geq 0.46\right)$. For the SPT developed in this work, a stable nematic phase is found over the whole range of the rigidity parameter (not shown for brevity). For intermediate-to-low values of the rigidity parameter, however, care must be taken with respect to the theoretical results. As we have shown in a recent Monte Carlo simulation study, ${ }^{27}$ rod-coil fluids of intermediate flexibility (for example 14-10, 10-8, or 8-6 rod-coils) experience a direct transition from the isotropic to a smectic phase. Therefore, to compare the theory to molecular simulation results, a description of the smectic phase would be required. Although it is possible to extent Onsager-like theories to describe smectic phases, ${ }^{28}$ this is beyond the scope of this work. For a value of the rigidity parameter equal (and probably close) to zero, the isotropic phase will experience a 
direct transition to the solid phase. ${ }^{66}$ An extension of the theory to the solid phase is also beyond the scope of this work.

\section{CONCLUSION}

An extension of Onsager's second virial theory is developed that analytically accounts for the effect of intramolecular flexibility on the isotropic-nematic phase behavior. The effect of chain flexibility on the second virial coefficient is described using an accurate, analytical approximation for the orientation-dependent pair-excluded volume. To approximate the higher order virial coefficients, both the Vega-Lago rescaling and Scaled Particle Theory (SPT) were evaluated.

Theoretical results for the equation of state and nematic order parameter were extensively tested against the results from MC simulations. For all systems considered, the results from the Vega-Lago rescaling and SPT are of similar accuracy. For linear chains, we found that both theories compare accurately to the MC data for chain lengths down to 9. For shorter chains, the isotropic-nematic transition is shifted to such high density that errors introduced by the approximation of the higher virial coefficients become apparent and, consequently, the pressure and density difference at the phase transition are under- and overestimated, respectively. It is important to note that the results for the Vega-Lago approach were obtained using an improved description of the isotropic EoS that was developed in a previous work. ${ }^{48}$ This new EoS explicitly treats the effect of intramolecular flexibility. When using isotropic equations of state for fully flexible chain fluids instead, the Vega-Lago approach was less successful. Although for many purposes, the isotropic phase behavior of linear and fully flexible chains can be assumed to be identical, ${ }^{39,67}$ the results obtained in this work show that there are differences that need to be captured to arrive at a satisfactory description of the isotropic-nematic phase equilibrium.

For rod-coil fluids, a near-quantitative agreement between theory and simulation data is obtained. The theoretical results correctly capture the general trends of the phase behavior with respect to the rigidity of the molecules. With increased rigidity, the shape anisotropy of the molecules is increased, resulting in a shift of the isotropic-nematic phase equilibrium to lower density. In addition, the relative density difference at the phase transition grows for increasingly rigid chains. Our results suggest that the approximate description of the higher virial coefficients that results from the VegaLago rescaling and SPT becomes less accurate for chains of increased flexibility.

\section{ACKNOWLEDGMENTS}

This research is supported by the Stichting voor Technische Wetenschappen (Dutch Technology Foundation, STW), applied science division of the Nederlandse organisatie voor Wetenschappelijk Onderzoek (Netherlands Organization for Scientific Research, NWO) and the Technology Program of the Ministry of Economic Affairs. In addition, this work was sponsored by the Stichting Nationale Computerfaciliteiten (National Computing Facilities Foundation, NCF) for the
TABLE I. The 8 model constants for the coefficients of the excluded volume expression given in Eqs. (A1)-(A6).

\begin{tabular}{lrrrrr}
\hline \hline $\mathrm{i}$ & $a_{1}$ & $a_{2 i}$ & $a_{3 i}$ & $b_{1}$ & $b_{2 i}$ \\
\hline 1 & 4.63 & -4.71 & 1.31 & 0.305 & -0.171 \\
2 & & 7.84 & -6.18 & & 3.32 \\
\hline \hline
\end{tabular}

use of supercomputing facilities, with financial support from NWO-EW.

\section{APPENDIX A: COEFFICIENTS FOR CALCULATING THE PAIR-EXCLUDED VOLUME}

In our previous work, ${ }^{43}$ we developed an accurate analytical approximation for the orientation-dependent pairexcluded volume of rod-coil molecules. The functional form of this approximation is given by Eq. (15) of the main text. The coefficients of Eq. (15) are given by

$$
\begin{gathered}
C_{1}\left(m, \chi_{\mathrm{R}}\right)=\frac{11 m-3}{m}+\frac{(m-1)^{2}}{m} \sum_{k=1}^{3} a_{k}\left(1-\chi_{\mathrm{R}}\right)^{k} \\
C_{2}\left(m, \chi_{\mathrm{R}}\right)=3.5339 \frac{(m-1)^{2}}{m} \chi_{\mathrm{R}}^{2} \\
C_{3}\left(m, \chi_{\mathrm{R}}\right)=\frac{(m-1)^{2}}{m} \sum_{k=1}^{2} b_{k}\left(1-\chi_{\mathrm{R}}\right)^{k}
\end{gathered}
$$

where the $a_{2}, a_{3}$, and $b_{2}$ parameters have an additional $m$ dependence as

$$
\begin{aligned}
& a_{2}=a_{21}+\frac{a_{22}}{m}, \\
& a_{3}=a_{31}+\frac{a_{32}}{m}, \\
& b_{2}=b_{21}+\frac{b_{22}}{m} .
\end{aligned}
$$

Here $a_{1}, a_{21}, a_{22}, a_{31}, a_{32}, b_{1}, b_{21}$, and $b_{22}$ are dimensionless constants that were adjusted to MC data of the excluded volume of pure rod-coil fluids. The values of these constants are listed in Table I.

\section{APPENDIX B: THE DECOUPLING APPROXIMATION}

In this appendix, the OVL theory is derived based on Parsons' decoupling approximation. ${ }^{29}$ Although originally Parsons considered rigid molecules (hard rods) only, his method is also perfectly valid for nonrigid chain molecules.

Assuming pairwise additive intermolecular interactions, the compressibility factor $Z=\beta P / \rho$ of an inhomogeneous system of anisotropic, nonrigid chain molecules can be obtained from the pressure equation as ${ }^{44}$

$$
\begin{aligned}
Z= & 1-\frac{\beta}{6 \rho V} \iiint r_{12} \frac{\partial \phi\left(\boldsymbol{r}_{12}, \tilde{\boldsymbol{\omega}}_{1}, \tilde{\boldsymbol{\omega}}_{2}\right)}{\partial r_{12}} g\left(\boldsymbol{r}_{12}, \tilde{\boldsymbol{\omega}}_{1}, \tilde{\boldsymbol{\omega}}_{2}\right) \rho\left(\tilde{\boldsymbol{r}}_{1}\right) \\
& \times \rho\left(\tilde{\boldsymbol{r}}_{2}\right) d \tilde{\boldsymbol{r}}_{1} d \tilde{\boldsymbol{r}}_{2} .
\end{aligned}
$$


Here, $g\left(\boldsymbol{r}_{12}, \tilde{\boldsymbol{\omega}}_{1}, \tilde{\boldsymbol{\omega}}_{2}\right)$ is the pair distribution function of molecules 1 and 2 . The intermolecular potential is defined as

$$
\phi\left(\boldsymbol{r}_{12}, \tilde{\boldsymbol{\omega}}_{1}, \tilde{\boldsymbol{\omega}}_{2}\right)= \begin{cases}\infty & \text { when } r_{12}<\sigma\left(\hat{\boldsymbol{r}}_{12}, \tilde{\boldsymbol{\omega}}_{1}, \tilde{\boldsymbol{\omega}}_{2}\right), \\ 0 & \text { when } r_{12} \geq \sigma\left(\hat{\boldsymbol{r}}_{12}, \tilde{\boldsymbol{\omega}}_{1}, \tilde{\boldsymbol{\omega}}_{2}\right),\end{cases}
$$

where $\sigma\left(\hat{\boldsymbol{r}}_{12}, \tilde{\boldsymbol{\omega}}_{1}, \tilde{\boldsymbol{\omega}}_{2}\right)$ is the conformation-dependent contact distance. This contact distance is a function of the conformations of molecules 1 and 2 and the orientational unit vector $\hat{\boldsymbol{r}}_{12}$ between the molecule's centers of mass.

Since we are concerned with describing nematic phases, the single-molecule density can be factorized into a uniform number density and a conformational distribution as $\rho(\tilde{\boldsymbol{r}})=\rho f(\tilde{\boldsymbol{\omega}})$ to obtain

$$
\begin{aligned}
Z= & 1-\frac{\beta}{6} \rho \iiint r_{12} \frac{\partial \phi\left(\boldsymbol{r}_{12}, \tilde{\boldsymbol{\omega}}_{1}, \tilde{\boldsymbol{\omega}}_{2}\right)}{\partial r_{12}} g\left(\boldsymbol{r}_{12}, \tilde{\boldsymbol{\omega}}_{1}, \tilde{\boldsymbol{\omega}}_{2}\right) f\left(\tilde{\boldsymbol{\omega}}_{1}\right) \\
& \times f\left(\tilde{\boldsymbol{\omega}}_{2}\right) d \boldsymbol{r}_{12} d \tilde{\boldsymbol{\omega}}_{1} d \tilde{\boldsymbol{\omega}}_{1} .
\end{aligned}
$$

The analogue of Onsager's original second virial theory for nonrigid molecules is obtained from the above equation by inserting the low-density limit of the pair distribution function and integrating the pressure over the volume. To extend the Onsager result to higher densities, Parsons used an improved approximation for the pair distribution function which is usually referred to as the "decoupling approximation." By writing both the intermolecular potential and the pair distribution function as a function of a reduced intermolecular separation $y=r_{12} / \sigma\left(\hat{\boldsymbol{r}}_{12}, \tilde{\boldsymbol{\omega}}_{1}, \tilde{\boldsymbol{\omega}}_{2}\right)$, all translational and conformational dependencies can be decoupled, according to

$$
\begin{aligned}
Z= & 1-\frac{\beta}{6} \rho \int y^{3} \frac{\partial \phi(y)}{\partial y} g(y) d y \\
& \times \iiint \sigma^{3} f\left(\tilde{\boldsymbol{\omega}}_{1}\right) f\left(\tilde{\boldsymbol{\omega}}_{2}\right) d \hat{\boldsymbol{r}}_{12} d \tilde{\boldsymbol{\omega}}_{1} d \tilde{\boldsymbol{\omega}}_{2} .
\end{aligned}
$$

The conformational part of this integral can be rewritten in terms of the pair-excluded volume, which is defined in Eq. (12), by writing

$$
\begin{aligned}
V_{\mathrm{ex}}\left(\tilde{\boldsymbol{\omega}}_{1}, \tilde{\boldsymbol{\omega}}_{2}\right) & =\int\left(1-\exp \left[-\beta \phi\left(\boldsymbol{r}_{12}, \tilde{\boldsymbol{\omega}}_{1}, \tilde{\boldsymbol{\omega}}_{2}\right)\right]\right) d \boldsymbol{r}_{12} \\
& =\int_{r_{12}=0}^{r_{12}=\sigma\left(\hat{\boldsymbol{r}}_{12}, \tilde{\boldsymbol{\omega}}_{1}, \tilde{\boldsymbol{\omega}}_{2}\right)} \int r_{12}^{2} d r_{12} d \hat{\boldsymbol{r}}_{12} \\
& =\frac{1}{3} \int \sigma\left(\hat{\boldsymbol{r}}_{12}, \tilde{\boldsymbol{\omega}}_{1}, \tilde{\boldsymbol{\omega}}_{2}\right)^{3} d \hat{\boldsymbol{r}}_{12} .
\end{aligned}
$$

To deal with the discontinuity of the intermolecular potential in the translational integral, a cavity correlation function $Y(y)$ $=g(y) \exp (\beta \phi)$ can be introduced as ${ }^{44}$

$$
\int y^{3} \frac{\partial \phi(y)}{\partial y} g(y) d y=-\frac{1}{\beta} \int y^{3} \frac{\partial \exp (-\beta \phi(y))}{\partial y} Y(y) d y .
$$

For the purely repulsive molecules considered in this work, the Boltzmann factor is a Heaviside step-function, the derivative of which is a Dirac delta function. Accordingly, the translational integral can be reduced to

$$
\int y^{3} \frac{\partial \phi(y)}{\partial y} g(y) d y=-\frac{1}{\beta} g\left(1^{+}\right) .
$$

Here, $g\left(1^{+}\right)$is the value of the pair distribution function in the limit of contact approaching from above (contact value theorem). ${ }^{44}$ Inserting Eqs. (B5) and (B7) into Eq. (B4), one obtains

$$
Z=1+\frac{1}{2} \rho g\left(1^{+}\right) \iiint V_{\mathrm{ex}}\left(\tilde{\boldsymbol{\omega}}_{1}, \tilde{\boldsymbol{\omega}}_{2}\right) f\left(\tilde{\boldsymbol{\omega}}_{1}\right) f\left(\tilde{\boldsymbol{\omega}}_{2}\right) d \tilde{\boldsymbol{\omega}}_{1} d \tilde{\boldsymbol{\omega}}_{2} .
$$

Using Eq. (11), this result can be recast in the form of a virial

$$
Z=1+g\left(1^{+}\right) B_{2}[f(\tilde{\boldsymbol{\omega}})] \rho .
$$

Finally, integration over density gives the residual Helmholtz energy

$$
\begin{aligned}
\frac{\beta A^{\mathrm{res}}}{N} & =\int \frac{Z-1}{\rho} d \rho \\
& =B_{2}[f(\tilde{\boldsymbol{\omega}})] \int g\left(1^{+}\right) d \rho .
\end{aligned}
$$

Let us now assume that $g\left(1^{+}\right)$of the system being described can be approximated by that of a reference system of the same molecular volume at the same packing fraction. Assuming the virial from Eq. (B9) is valid for the given reference system (note that this is only exact for a system of hard spheres), we can write

$$
g\left(1^{+}\right) \approx g_{\mathrm{ref}}\left(1^{+}\right)=\left(\frac{Z_{\mathrm{ref}}-1}{\rho}\right) \frac{1}{B_{2, \mathrm{ref}}} .
$$

Substitution in Eq. (B9) and integrating the compressibility leads to

$$
\frac{\beta A^{\mathrm{res}}}{N}=\frac{\beta A_{\mathrm{ref}}^{\mathrm{res}}}{N} \frac{B_{2}[f(\tilde{\boldsymbol{\omega}})]}{B_{2, \mathrm{ref}}}
$$

\section{APPENDIX C: SOLUTION OF EQS. (6) AND (11) IN TERMS OF THE OTF}

In this appendix, we summarize the solution of Eqs. (6) and (11) in terms of the OTF. The resulting equations are expressed solely in terms of the degree of orientational order $\alpha$. For details on the derivations, the reader is referred to the work of Franco-Melgar et al. ${ }^{12,68}$ The solution of Eqs. (6) and (11) in terms of the OTF can be derived as, respectively

$$
\frac{\beta A_{\text {aniso }}^{\text {id }}}{N}=\ln [\alpha \operatorname{coth}(\alpha)]-1+\frac{\arctan (\sinh (\alpha))}{\sinh (\alpha)}
$$

and

$$
\begin{aligned}
B_{2, \text { nem }}= & \frac{C_{1}}{2}+C_{2} \frac{\pi I_{2}(2 \alpha)}{4 \sinh ^{2}(\alpha)} \\
& +\frac{C_{3}}{2 \sinh ^{2}(\alpha)}\left\{\sinh (2 \alpha)\left[\frac{2}{\alpha}+\frac{6}{\alpha^{3}}\right]+\right. \\
& \left.-\cosh (2 \alpha)\left[\frac{5}{\alpha^{2}}+\frac{3}{\alpha^{4}}\right]-\frac{1}{\alpha^{2}}+\frac{3}{\alpha^{4}}\right\} .
\end{aligned}
$$

Here, $C_{1}, C_{2}$, and $C_{3}$ are the coefficients of the excluded volume expression from Eq. (15). The quantity $I_{2}(2 \alpha)$ is a modified Bessel function of second order, which is defined by the 
following general integral representation:

$$
I_{2}(2 \alpha)=\frac{1}{\pi} \int_{u=0}^{\pi} \exp (2 \alpha \cos u) \cos (2 u) d u .
$$

The Bessel function is solved by numerical integration. The derivatives of Eqs. $(\mathrm{C} 1)$ and $(\mathrm{C} 2)$ to $\alpha$, which are required to solve Eq. (30) for $\alpha=\alpha_{\text {eq }}$, can be derived as

$$
\left(\frac{\partial\left[\beta A_{\text {aniso }}^{\mathrm{id}} / N\right]}{\partial \alpha}\right)_{N V T}=\frac{1}{\alpha}+\frac{\arctan [\sinh (\alpha)] \cosh (\alpha)}{\sinh ^{2}(\alpha)},
$$

$$
\begin{aligned}
& \left(\frac{\partial B_{2, \text { nem }}}{\partial \alpha}\right)_{N V T} \\
& =\frac{C_{2} \pi}{2 \sinh ^{2}(\alpha)}\left\{I_{2}(2 \alpha)\left[\frac{\alpha^{2}+3}{3 \alpha}-\operatorname{coth}(\alpha)\right]-\frac{\alpha}{3} I_{4}(2 \alpha)\right\} \\
& \quad+\frac{C_{3}}{\sinh ^{2}(\alpha)}\left\{\cosh (2 \alpha)\left(\frac{2}{\alpha}+\frac{11}{\alpha^{3}}+\frac{6}{\alpha^{5}}\right)\right. \\
& \quad-\sinh (2 \alpha)\left(\frac{6}{\alpha^{2}}+\frac{12}{\alpha^{4}}\right) \\
& \quad+\frac{1}{\alpha^{3}}-\frac{6}{\alpha^{5}}-\operatorname{coth}(\alpha)\left[\sinh (2 \alpha)\left(\frac{2}{\alpha}+\frac{6}{\alpha^{3}}\right)-\cosh (2 \alpha)\right. \\
& \left.\left.\quad \times\left(\frac{5}{\alpha^{2}}+\frac{3}{\alpha^{4}}\right)-\frac{1}{\alpha^{2}}+\frac{3}{\alpha^{4}}\right]\right\} .
\end{aligned}
$$

Once a value for $\alpha_{\text {eq }}$ is obtained, the nematic order parameter can be calculated from Eq. (31).

${ }^{1}$ S. Chandrasekhar, Liquid Crystals, 2nd ed. (Cambridge University Press, Cambridge, 1992).

${ }^{2}$ G. W. Gray, Thermotropic Liquid Crystals (John Wiley \& Sons, Chichester, 1987), Chap. 2

${ }^{3}$ H. Zocher, Z. Anorg. Chem. 147, 91 (1925).

${ }^{4}$ I. Langmuir, J. Chem. Phys. 6, 873 (1938).

${ }^{5}$ F. C. Bawden, N. W. Pirie, J. D. Bernal, and I. Frankuchen, Nature (London) 138, 1051 (1936).

${ }^{6}$ P. G. de Gennes and J. Prost, The Physics of Liquid Crystals, 2nd ed. (Oxford University Press, Oxford, 1993).

${ }^{7}$ P. J. Collins, Liquid Crystals: Nature's Delicate Phase of Matter, 2nd ed. (Princeton University Press, Princeton, 2002).

${ }^{8}$ J. Gross and P. J. Jansens, patent application WO2008147181A1/NL2000654-C2 (4 December 2008).

${ }^{9}$ M. de Groen, T. J. H. Vlugt, and T. W. de Loos, J. Phys. Chem. B 116, 9101 (2012).

${ }^{10}$ L. Onsager, Phys. Rev. 62, 558 (1942).

${ }^{11}$ L. Onsager, Ann. N.Y. Acad. Sci. 51, 627 (1949).

${ }^{12}$ M. Franco-Melgar, A. J. Haslam, and G. Jackson, Mol. Phys. 106, 649 (2008).

${ }^{13}$ P. Bolhuis and D. Frenkel, J. Chem. Phys. 106, 666 (1997).

${ }^{14}$ D. Frenkel and R. Eppenga, Phys. Rev. Lett. 49, 1089 (1982).

${ }^{15}$ R. Eppenga and D. Frenkel, Mol. Phys. 52, 1303 (1984).

${ }^{16}$ X. Wen, R. B. Meyer, and D. L. D. Caspar, Phys. Rev. Lett. 63, 2760 (1989)

${ }^{17}$ S. D. Zhang, P. A. Reynolds, and J. S. Duijneveldt, J. Chem. Phys. 117, 9947 (2002)

${ }^{18}$ L. Wu, H. H. Wensink, G. Jackson, and E. A. Müller, Mol. Phys. 110, 1269 (2012).
${ }^{19}$ S. C. McGrother, D. C. Williamson, and G. Jackson, J. Chem. Phys. 104, 6755 (1996).

${ }^{20}$ D. Frenkel and B. M. Mulder, Mol. Phys. 55, 1171 (1985).

${ }^{21}$ A. Samborski, G. T. Evans, C. P. Mason, and M. P. Allen, Mol. Phys. 81, 263 (1994).

${ }^{22}$ E. de Miguel and E. M. del Río, J. Chem. Phys. 118, 1852 (2003).

${ }^{23}$ H. Fynewever and A. Yethiraj, J. Chem. Phys. 108, 1636 (1998).

${ }^{24}$ D. C. Williamson and G. Jackson, J. Chem. Phys. 108, 10294 (1998).

${ }^{25}$ C. McBride, C. Vega, and L. G. MacDowell, Phys. Rev. E 64, 011703 (2001).

${ }^{26}$ C. McBride and C. Vega, J. Chem. Phys. 117, 10370 (2002).

${ }^{27}$ B. Oyarzún, T. van Westen, and T. J. H. Vlugt, J. Chem. Phys. 138, 204905 (2013).

${ }^{28}$ M. Esposito and G. T. Evans, Mol. Phys. 83, 835 (1994).

${ }^{29}$ J. Parsons, Phys. Rev. A 19, 1225 (1979).

${ }^{30}$ S. D. Lee, J. Chem. Phys. 87, 4972 (1987).

${ }^{31}$ S. D. Lee, J. Chem. Phys. 89, 7036 (1988).

${ }^{32}$ C. Vega and S. Lago, J. Chem. Phys. 100, 6727 (1994).

${ }^{33}$ M. A. Cotter and D. E. Martire, J. Chem. Phys. 52, 1902 (1970).

${ }^{34}$ M. A. Cotter, Phys. Rev. A 10, 625 (1974).

${ }^{35}$ M. A. Cotter, J. Chem. Phys. 66, 1098 (1977).

${ }^{36}$ T. Boublík and I. Nezbeda, Chem. Phys. Lett. 46, 315 (1977).

${ }^{37}$ T. Boublík, Mol. Phys. 44, 1369 (1981).

${ }^{38}$ T. Boublík, Mol. Phys. 68, 191 (1989).

${ }^{39}$ T. Boublík, C. Vega, and M. Diaz-Penã, J. Chem. Phys. 93, 730 (1990).

${ }^{40}$ T. Jiang and J. Wu, J. Chem. Phys. 127, 034902 (2007).

${ }^{41}$ P. J. Flory, Proc. R. Soc. London, Ser. A 234, 60 (1956)

${ }^{42}$ J. S. van Duijneveldt and M. P. Allen, Mol. Phys. 92, 855 (1997).

${ }^{43}$ T. van Westen, T. J. H. Vlugt, and J. Gross, J. Chem. Phys. 137, 044906 (2012).

${ }^{44}$ J. P. Hansen and I. R. McDonald, Theory of Simple Liquids, 3rd ed. (Academic Press, London, 2006).

${ }^{45}$ M. Franco-Melgar, A. J. Haslam, and G. Jackson, Mol. Phys. 107, 2329 (2009)

${ }^{46}$ T. Hino and J. M. Prausnitz, Polymer 40, 1241 (1999).

${ }^{47}$ H. Liu and Y. Hu, Fluid Phase Equilib. 122, 75 (1996)

${ }^{48}$ T. van Westen, B. Oyarzún, T. J. H. Vlugt, and J. Gross, "An equation of state for the isotropic phase of linear, partially flexible and fully flexible tangent hard-sphere chain fluids," Mol. Phys. (2013).

${ }^{49}$ D. Chandler and L. R. Pratt, J. Chem. Phys. 65, 2925 (1976).

${ }^{50}$ L. R. Pratt and D. Chandler, J. Chem. Phys. 66, 147 (1977).

${ }^{51}$ C. McBride, M. Wilson, and J. Howard, Mol. Phys. 93, 955 (1998).

${ }^{52}$ W. G. Chapman, G. Jackson, and K. E. Gubbins, Mol. Phys. 65, 1057 (1988).

${ }^{53}$ J. Gross and G. Sadowski, Ind. Eng. Chem. Res. 40, 1244 (2001).

${ }^{54}$ M. G. Martin and J. I. Siepmann, J. Phys. Chem. B 102, 2569 (1998).

${ }^{55}$ J. E. Mayer and M. G. Mayer, Statistical Mechanics, 7th ed. (John Wiley \& Sons, New York, 1940), Chap. 13.

${ }^{56}$ S. I. Sandler, An Introduction to Applied Statistical Thermodynamics (Wiley, Hoboken, 2011).

${ }^{57}$ D. C. Williamson and G. Jackson, Mol. Phys. 86, 819 (1995).

${ }^{58}$ K. M. Jaffer, S. B. Opps, D. E. Sullivan, B. G. Nickel, and L. Mederos, J. Chem. Phys. 114, 3314 (2001).

${ }^{59}$ A. Isihara, J. Chem. Phys. 19, 1142 (1951).

${ }^{60}$ G. Lasher, J. Chem. Phys. 53, 4141 (1970).

${ }^{61}$ J. Herzfeld, A. E. Berger, and J. W. Wingate, Macromolecules 17, 1718 (1984).

${ }^{62}$ H. N. W. Lekkerkerker, P. Coulon, R. Van Der Haegen, and R. Deblieck, J. Chem. Phys. 80, 3427 (1984).

${ }^{63}$ S. Lee and R. B. Meyer, J. Chem. Phys. 84, 3443 (1986).

${ }^{64}$ D. Williamson and G. Jackson, Mol. Phys. 83, 603 (1994)

${ }^{65}$ C. Vega, C. McBride, and L. G. MacDowell, J. Chem. Phys. 115, 4203 (2001).

${ }^{66}$ F. Alavi and F. Feyzi, J. Chem. Phys. 138, 024903 (2013).

${ }^{67}$ F. J. Blas, E. Sanz, C. Vega, and A. Galindo, J. Chem. Phys. 119, 10958 (2003).

${ }^{68}$ M. Franco-Melgar, A. J. Haslam, and G. Jackson, Mol. Phys. 110, 3107 (2012). 